\title{
PREDICATIVE FREGE ARITHMETIC AND 'EVERYDAY' MATHEMATICS
}

\author{
RICHARD G HECK JR
}

The primary purpose of this note is to demonstrate that predicative Frege arithmetic naturally interprets certain weak but non-trivial arithmetical theories. It will take almost as long to explain what this means and why it matters as it will to prove the results.

\section{Fregean Arithmetics}

The sole non-logical axiom of Fregean arithmetics is the principle generally known as HP: ${ }^{1}$

$$
\begin{aligned}
\mathrm{N} x: A(x)=\mathrm{N} x: B(x) \equiv \exists R[ & \forall x \forall y \forall z \forall w(R x y \wedge R z w \rightarrow x=z \equiv y=w) \wedge \\
& \forall x(A(x) \rightarrow \exists y(R x y \wedge B(y))) \wedge \\
& \forall y(B(y) \rightarrow \exists x(R x y \wedge A(x)))]
\end{aligned}
$$

HP asserts that the number of $A \mathrm{~s}$ is the same as the number of $B \mathrm{~s}$ just in case the $A$ s can be correlated one-one with the $B$ s. Fregean arithmetics are thus theories of cardinal numbers.

Since HP quantifies over relations, the logic of a Fregean arithmetic has to be some sort of second-order logic, and how much arithmetic we can interpret will depend upon how strong that logic is. Now, the strength of second-order logic derives from the so-called comprehension axioms, ${ }^{2}$ each of which states, in effect, that a given formula defines a 'concept' or a 'relation': something in the domain of the second-order variables. These axioms take the form:

$$
\begin{aligned}
\exists F \forall x[F x & \equiv A(x)] \\
\exists F \forall x \forall y[F x y & \equiv A(x, y)]
\end{aligned}
$$

and similarly for formulae of increasing arity. The weakest second-order logic, which has no such axioms, adds essentially nothing to first-order logic. At the other extreme, we have 'full' second-order logic, which has

\footnotetext{
${ }^{1}$ Here ' $\mathrm{N} x: \phi x$ ' is a 'variable binding term-forming operator' (vbto): It attaches to an open formula to create a term. An alternative formulation uses a functor '\#' that attaches only to variables, thus: \#F. I prefer to use vbtos because it makes the definitions and actual argumentation much simpler. The results proven here do not, however, seem to depend upon this choice (though there are other results that do depend upon it). I'll continue to confine remarks about this matter to the footnotes.

2 There are other axioms one can also consider, such as various choice principles, but these will not play a role here.
} 
comprehension for every formula in which ' $F$ ' does not occur free. Systems of intermediate strength place various restrictions on the formulae that can appear on the right-hand side. Thus, 'predicative' second-order logic has comprehension only for formulae containing no bound secondorder variables, and $\Pi_{1}^{1}$ second-order logic has comprehension only for $\Pi_{1}^{1}$ formulae, that is, for formulae of the form $\forall F \cdots \forall G \phi$, where $\phi$ contains no second-order quantifiers.

Full Frege arithmetic (FA) is full second-order logic plus HP. As is now well-known, it is strong enough to interpret full second-order arithmetic, or 'analysis'. ${ }^{3}$ But the proofs of the key results here-the existence of successors, sums, and products-require only $\Pi_{1}^{1}$ comprehension (Heck, 2011a), so $\Pi_{1}^{1}-F A$ interprets $\Pi_{1}^{1}-P A$, and, indeed, the parallel extends up through the analytical hierarchy: $\Pi_{n}^{1}$-FA interprets $\Pi_{n}^{1}$-PA. The converse is also true: $\Pi_{n}^{1}$-PA interprets $\Pi_{n}^{1}$-FA (Linnebo, 2004). We get just as much induction as we are prepared to buy with comprehension.

\section{INTERPRETATIONS, NATURAL AND UNNATURAL}

When I speak of 'interpretation' here, I mean what Tarski, Mostowski, and Robinson (1953) called 'relative interpretation'. ${ }^{4}$ A relative interpretation of a target theory $\mathcal{T}$ into a base theory $\mathcal{B}$ is based upon a translation from the language of $\mathcal{T}$ into that of $\mathcal{B}$ : of $\mathcal{L}_{\mathcal{T}}$ into $\mathcal{L}_{\mathcal{B}}$. The translation consists of two parts: (i) 'definitions' of the primitives of $\mathcal{L}_{\mathcal{T}}$ in terms of those of $\mathcal{L}_{\mathcal{B}}$; and (ii) a specification of a 'domain' by some formula $\delta(x)$ of $\mathcal{L}_{\mathcal{B}}$. These induce a translation from formulae of $\mathcal{L}_{\mathcal{T}}$ to those of $\mathcal{L}_{\mathcal{B}}$ : The primitives are translated as specified by (i), and the rest happens compositionally, with quantifiers being 'relativized' to $\delta(x)$, so that $\forall x(\phi(x))$ becomes: $\forall x\left(\delta(x) \rightarrow \phi^{t} x\right)$, where $\phi^{t}(x)$ is the translation of $\phi(x)$. Such a translation supports a relative interpretation if, under this translation, axioms of $\mathcal{T}$ go over to theorems of $\mathcal{B},{ }^{5}$ so that theorems of $\mathcal{T}$ also go over into theorems of $\mathcal{B} .^{6}$ It then follows, among other things, that, if $\mathcal{B}$ is consistent, so is $\mathcal{T}$.

The interpretation of analysis in full FA is 'natural' in the sense that the 'definitions' used in the translation are themselves 'natural': that is, they have some reasonable claim actually to count as definitions in

\footnotetext{
${ }^{3}$ This was first noted, though without proof, by Allen Hazen (1985), in his review of Frege's Conception of Numbers as Objects (Wright, 1983). Boolos (1998a) was the first to publish a proof, in 1987. An improved version can be found in the second appendix of the paper he and I wrote together (Boolos and Heck, 1998). That appendix is due entirely to Boolos.

${ }^{4}$ Visser $(2009 \mathrm{~b}, \S 2)$ gives a precise account of what an interpretation is. He also considers much more sophisticated notions of interpretation than we will need, but part of the point here is precisely that we do not need to use anything very sophisticated.

${ }^{5}$ We also need $\mathcal{B}$ to prove that the domain is non-empty and, if there are constants and function-symbols, to prove closure conditions with respect to the domain.

${ }^{6}$ This is because of the 'compositional' nature of the translation.
} 
something other than a purely formal sense. For example, the usual 'definition' of ' 0 ' is:

$$
0 \stackrel{d f}{=} \mathrm{N} x:(x \neq x)
$$

which seems pretty reasonable. The definitions of predecession and of the concept of a natural number, which we shall consider shortly, are more controversial, but they still have at least some claim to be 'natural' in this sense. Of course, there is in general no requirement that the translation on which a relative interpretation is based should be 'natural', and there are plenty of cases where it is not. One familiar example would be the interpretation of syntax in arithmetic via Gödel numbering.

Our interest here is in predicative FA, and the goal is to show that we can naturally interpret certain weak but non-trivial arithmetical theories in it. If we do not restrict ourselves to 'natural' interpretations, then this result does not now need proving. John P. Burgess (2005, ch. 2) has already shown that predicative FA interprets Robinson arithmetic and so, by well-established results, interprets stronger theories still, such as $\mathrm{I} \Delta_{0} \cdot{ }^{7}$ From a philosophical point of view, however, there might seem to be an element of cheating in Burgess's argument. Consider, for example, the proof that every number has a successor. If we define predecession and the concept of a natural number in the usual way: ${ }^{8}$

$$
\begin{aligned}
\mathrm{P} m n & \stackrel{d f}{\equiv} \exists F \exists y[n=\mathrm{N} x: F x \wedge F y \wedge m=\mathrm{N} x:(F x \wedge x \neq y)] \\
\mathbb{N} n & \stackrel{d f}{=} \forall F[F 0 \wedge \forall x(F x \wedge P x y \rightarrow F y) \rightarrow F n]
\end{aligned}
$$

then, as Linnebo (2004) shows, we cannot prove that every natural number has a successor. The obvious sort of argument by induction fails, because the induction would have to be on $\exists y(\mathrm{P} \xi y)$. Since this formula contains a second-order quantifier (in the definition of $P$ ), we do not have comprehension for it in predicative FA and therefore do not have induction for it either. We can still interpret a theory that proves that successors exist by changing our definition of what a natural number is, in effect, restricting the natural numbers to the numbers that do have successors. ${ }^{9}$ But, as Linnebo $(2004$, p. 172) notes, “... it would be exceedingly implausible to claim that anything like [this] notion of a natural [number] is involved in our ordinary arithmetical knowledge".

\footnotetext{
${ }^{7}$ Results closer to the one proven here have been obtained by Visser (2009b; 2011), but he is not concerned with 'natural' interpretations, either.

${ }^{8}$ Linnebo (2004, pp. 172-3) raises questions about whether Frege's definiton of predecession is 'natural' in the sense at issue here. His worries concern the logical complexity of the definition: the fact that it is $\Sigma_{1}^{1}$. I have argued elsewhere, however, that its complexity is something of an illusion (Heck, 2011d, §12.1) and so shall not discuss the matter here.

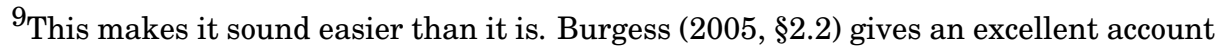
of the details of this kind of construction, known as the shortening of cuts, which is originally due to Robert Solovay.
} 
Something similar happens in the cases of addition and multiplication. Addition can be defined in the following 'natural' way: ${ }^{10}$

$$
\begin{aligned}
\mathrm{A}(a, b, c) \stackrel{d f}{=} \exists F \exists G[a=\mathrm{N} x: F x \wedge b=\mathrm{N} x: G x \wedge \\
\neg \exists x(F x \wedge G x) \wedge c=\mathrm{N} x:(F x \vee G x)]
\end{aligned}
$$

Sums thus reflect the cardinalities of disjoint unions. Again, however, we have no hope of showing that every pair of natural numbers has a sum that is also a natural number, or even that sums always exist. The only strategy that suggests itself is to argue by induction, and the restriction to predicative comprehension will again frustrate the attempt. As before, we can still interpret a theory that says that every pair of natural numbers has a sum by changing our definition of what a natural number is, in effect, simply restricting the domain to the numbers that $d o$ have sums. Surely, however, our concept of natural number does not include a clause specially restricting it to numbers that have sums and products.

Burgess's interpretation of $Q$ in predicative FA is thus not 'natural' in the sense isolated above. ${ }^{11}$ (Not that he intended it to be.) The definitions of zero, predecession, addition, and multiplication (see below) are 'natural' enough. But the definition of natural number is not 'natural' at all. Rather, the definition has been gerrymandered to give us what we want.

As it happens, there is a way around these problems in the case of successor (Heck, 2011e), but, so far as I can see, there is not much hope that there will be a way around them in the case of addition and multiplication. ${ }^{12}$ Prior to the work reported below, therefore, the strongest mathematical theory we knew could 'naturally' be interpreted in a predicative version of FA was the simple theory of successor:

\footnotetext{
${ }^{10}$ If we want to work with the functor formulation, then this needs to be rephrased, since in many cases below we will not have comprehension for disjunctions. One way to rephrase it is:

$$
\begin{gathered}
\mathrm{A}(a, b, c) \stackrel{d f}{=} \exists F \exists G \exists H[a=\mathrm{N} x: F x \wedge b=\mathrm{N} x: G x \wedge c=\mathrm{N} x: H x \wedge \\
\neg \exists x(F x \wedge G x) \wedge \forall x(F x \vee G x \equiv H x)]
\end{gathered}
$$

It is precisely this sort of indirection that leads me to prefer the formulation with a vbto, at least in contexts like this one, where we are talking about 'natural' definitions and interpretations.

${ }^{11}$ Visser suggests that "it would be interesting to see whether there is a coherent foundational idea corresponding to the methodology of shortening cuts". I agree, but at present we have, as he also says, "no hint what such an idea could look like" (Visser, 2011, p. 116).

${ }^{12}$ I do not actually have a proof that existence of sums cannot be proven in ramified predicative FA, but I suspect that the model Linnebo uses to show that existence of successor cannot be proven in (simple) predicative FA can be used to establish this conclusion, since that model presumably shows that even $n+1$ does not always exist.
} 
(1) $\neg \exists x(\mathbb{N} x \wedge \mathrm{P} x 0)$

(2) $\forall x \forall y \forall z(\mathrm{P} x z \wedge \mathrm{P} y z \rightarrow x=y)$

(3) $\forall x \forall y \forall z(\mathrm{P} x y \wedge \mathrm{P} x z \rightarrow y=z)$

(4) $\forall x(\mathbb{N} x \rightarrow \exists y(\mathbb{N} y \wedge \mathrm{P} x y))$

But this theory is trivial, in the sense that it is decidable (or, at least, has a decidable extension). Until recently, then, we did not know how naturally to interpret any non-trivial arithmetical theory in a predicative form of FA. My primary purpose here is to remedy this situation by showing that there are mathematical theories that can be naturally interpreted in predicative FA and that are non-trivial, in the sense that they are subject to Gödel's first incompleteness theorem and so are essentially undecidable.

Why care about whether the interpretation is 'natural'? Investigations of FA have generally been driven not by purely technical concerns but by philosophical ones. FA arrived on the philosophical scene when Wright (1983) suggested that it might support a reformed logicism according to which the basic truths of arithmetic are, though not logical truths, still analytic or conceptual truths, since they are logical consequences of HP, which is itself an analytic or conceptual truth. There is much to debate here. ${ }^{13}$ But the present point is simply that any claim of this form needs there to be some close relationship between the technical definitions and the intuitive notions that are their inspiration. I do not mean, then, in any way to disparage 'unnatural' interpretations, but only to note that there is a kind of philosophical significance one might want the fact that one theory can be interpreted in another to have, a kind of significance that an interpretation cannot have, unless the definitions on which it is based are 'natural'.

It has even been suggested that HP may be of some psychological significance, that HP may, as a matter of empirical fact, play a foundational role in our ordinary mathematical knowledge (Decock, 2008; Pietroski and Lidz, 2008; Heck, 2011a). It should be clear that, if mathematical work of the sort undertaken here is to throw any light at all on the psychology of arithmetic, it is especially important that the interpretations we consider should be 'natural', in the sense that they are faithful to the content of everyday mathematical notions.

Of course, the notion of a natural interpretation is not likely to be subject to scientific explication, not in our present state of knowledge. For the time being, then, it remains a philosophical notion, so judgements about what is 'natural' will be controversial and defeasible, as

\footnotetext{
${ }^{13}$ The exchange between Wright (2001) and Boolos (1998b) is a good place to start. See my book Frege's Theorem (Heck, 2011c) for my own explorations.
} 
philosophical judgements tend to be. If we had a general theory of concepts and their contents, we could presumably do better, since the notion of naturalness could then be recast in psychological or semantic terms, and the question whether some interpretation was 'natural' might then become an empirical one. But we do not now have such a theory, not one that commands much agreement, anyway. For the time being, then, we are stuck doing philosophy.

There is another point in the same vicinity. Linnebo (2004, p. 168) argues that, “... if Frege's Theorem is to have this sort of philosophical significance, ... its proof must have at least a reasonable claim to being just an explication of our ordinary arithmetical reasoning". That is: The proofs themselves must, in a related sense, also be 'natural'. I am sympathetic (Heck, 2011a, pp. 156-60; 2011b, §11.2)—though I am not as sure as Linnebo is that Frege's proof of the existence of successors cannot claim this title. ${ }^{14}$ But the proofs to be given below, I think, pretty clearly can. It does not follow, of course, that the results proven here really do have the sort of significance that some of us have thought Frege's Theorem might have. (Naturalness is a necessary condition, not a sufficient one.) What does follow is that at least some of the objections that have been brought against such views simply do not arise if we limit our attention to what we might call 'everyday' arithmetic. These include the objections, originating with Poincaré, to Frege's definition of natural number, since no appeal to the concept of natural number will be made in what follows. ${ }^{15}$ They also include Linnebo's worries about the proof that successors exist, and about the inability of predicative FA to deliver the existence of sums and products, since, as we shall see, plenty of mathematics is available without these claims. I'll return to this topic in the final section.

\section{ExCeedingly Weak Arithmetics}

As said, I aim to show that there are non-trivial arithmetical theories that can be 'naturally' interpreted in predicative FA. That this might be possible emerges from the following observation. Although predicative FA does not prove that all natural numbers have successors, given the

\footnotetext{
${ }^{14}$ The underlying idea is simply that every natural number is the number of its predecessors, i.e., $n=\mathrm{N} x:(x<n)$, and this can be given a fairly simple, intuitive proof that appeals only to basic facts about predecession and its relation to $<$. Indeed, such a proof is given below for each individual case. An even better version might begin with $n=\mathrm{N} x:(1 \leq x \wedge x \leq n)$, which is one of the basic facts about counting, and then proceed to observe that $\mathrm{N} x:(0 \leq x \wedge x \leq n)$ will therefore be one more than $n$. Turning either of these into a rigorous proof takes work, no doubt, but that is mostly because of the role played by the ancestral in relating $<$ to predecession.

${ }^{15}$ As it happens, I think this objection can also be met, since I think it is possible to give a 'natural' definition of the ancestral: one that has some claim (as Frege's does not) to being intensionally correct (Heck, 2012, §7.7).
} 
'natural' definition of that notion, it does prove that each natural number has a successor. And, although it does not prove that all pairs of natural numbers have sums, given the 'natural' definition of that notion, it does prove that each pair of natural numbers has a sum. In fact, predicative FA proves that the sum is what one would expect it to be. Similarly for multiplication. That makes it plausible that predicative FA might naturally interpret a version of the theory Tarski, Mostowski, and Robinson (1953, pp. 52-3) called 'R'.

The language of $R$ is $\{0, S,+, \times\}$, and it has as axioms all instances of the following five schemes: ${ }^{16}$

$$
\begin{aligned}
& \bar{n}+\bar{m}=\overline{n+m} \\
& \bar{n} \times \bar{m}=\overline{n \times m} \\
& \bar{n} \neq \bar{m}, \text { whenever } n \neq m \\
& x \leq \bar{n} \equiv x=\overline{0} \vee \cdots \vee x=\bar{n} \\
& x \leq \bar{n} \vee \bar{n} \leq x
\end{aligned}
$$

Here, $\bar{n}$ is the numeral for $n$, defined in the usual way:

$$
\begin{gathered}
\overline{0} \stackrel{d f}{=} \text { ' } 0 \text { ' } \\
\overline{n+1} \stackrel{d f}{=} \text { ' } \mathrm{S} \text { ' } \frown \bar{n}
\end{gathered}
$$

As for $\leq$, we can take it to be defined in the usual way as well:

$$
x \leq y \stackrel{d f}{=} \exists z(z+x=y)
$$

It is also possible to treat $\leq$ as primitive, governed by the preceding as an axiom, and one can even treat $\leq$ as primitive as as governed only by (R4) and (R5) as axioms. This difference will not much matter for our purposes, though I shall make some remarks on it in the footnotes.

Though $R$ is in many ways obviously very weak, there are other ways in which it is surprisingly strong. ${ }^{17}$ All recursive functions are 'numeralwise representable' in $\mathrm{R}$. That is, given any recursive function $\phi\left(x_{1}, \ldots, x_{k}\right)$, we can find a formula $\Phi\left(x_{1}, \ldots, x_{k}, y\right)$ such that $\mathrm{R}$ proves

$$
\Phi\left(\overline{n_{1}}, \ldots, \overline{n_{k}}, y\right) \equiv y=\bar{m}
$$

whenever $\phi\left(n_{1}, \ldots, n_{k}\right)$ is indeed $m$ (Tarski et al., 1953, pp. 56ff). This is enough for Gödel's first incompleteness theorem, so R is essentially undecidable (Tarski et al., 1953, pp. 60-61). Moreover, $\mathrm{R}$ is $\Sigma_{1}$ complete: Every true $\Sigma_{1}$ sentence is provable in $R$.

\footnotetext{
${ }^{16}$ So R has infinitely many axioms corresponding to each of the schemes.

${ }^{17}$ There are other reasons that $R$ is interesting, too. Indeed, it was Visser's fascinating paper "Why the Theory R Is Special" (Visser, 2009c) that got me thinking about the present topic.
} 
Now, it is obvious that we are not going to be able to interpret $R$ itself in predicative Frege arithmetic in any 'natural' way. This is because the language of $R$ features function symbols $S,+$, and $\times$, and so the following are logical axioms of $\mathrm{R}$ :

$$
\begin{gathered}
\forall x \exists y(\mathrm{~S} x=y) \\
\forall x \forall y \exists z(x+y=z) \\
\forall x \forall y \exists z(x \times y=z)
\end{gathered}
$$

The whole problem here is that we cannot prove the existence of successors, sums, and products given the 'natural' definitions of these notions (and of the concept of natural number), so we are not going to prove (translations of) these logical axioms, so we are not going to interpret $R$ using those definitions.

Function-symbols are often inconvenient, for this reason: They mask existence and uniqueness assumptions and, worse, build them into the logic. ${ }^{18}$ We can make these assumptions explicit by reformulating $R$ in a purely relational language, thus arriving at the purely relational theory $\mathrm{R}^{*}$ with the following axiom schemes:

$\begin{array}{ll}\text { (R0e) } & \forall x \exists y(\mathrm{P}(x, y)) \\ \text { (R0u) } & \forall x \forall y \forall z(\mathrm{P}(x, y) \wedge \mathrm{P}(x, z) \rightarrow y=z)] \\ \text { (R1e) } & \forall x \forall y \exists z(\mathrm{~A}(x, y, z)) \\ \text { (R1u) } & \forall x \forall y \forall z \forall w(\mathrm{~A}(x, y, z) \wedge \mathrm{A}(x, y, w) \rightarrow z=w) \\ \text { (R1*) } & \mathrm{A}(\bar{n}, \bar{m}, \overline{n+m}) \\ \text { (R2e) } & \forall x \forall y \exists z(\mathrm{M}(x, y, z)) \\ \text { (R2u) } & \forall x \forall y \forall z \forall w(\mathrm{M}(x, y, z) \wedge \mathrm{M}(x, y, w) \rightarrow z=w) \\ \text { (R2*) } & \mathrm{M}(\bar{n}, \bar{m}, \overline{n \times m}) \\ \text { (R3*) } & \bar{n} \neq \bar{m}, \text { whenever } n \neq m \\ \text { (R4*) } & x \leq \bar{n} \equiv x=\overline{0} \vee \cdots \vee x=\bar{n} \\ \text { (R5*) } & x \leq \bar{n} \vee \bar{n} \leq x\end{array}$

Here, $\mathrm{P}, \mathrm{A}$, and $\mathrm{M}$ are relational substitutes for $\mathrm{S},+$, and $\times$, and so $\leq$ is defined as: $x \leq y \stackrel{d f}{=} \exists z(\mathrm{~A}(z, x, y))$. Since we do not have $\mathrm{S}$, the numerals also have to be redefined. We do this contextually, using Russell's theory

\footnotetext{
${ }^{18}$ Boolos used frequently to make this very complaint about the cardinality operator $\mathrm{N} x: \phi x$. Since it is a convention of logic that function symbols denote total functions, the mere use of this expression involves assumptions of existence and uniqueness. The formulation of HP that he called "Numbers" (Boolos, 1998a, p. 186) is thus analogous to what we are doing here.
} 
of descriptions: ${ }^{19}$

$$
\begin{aligned}
& \overline{0} \stackrel{d f}{=} 0 \\
& \overline{1} \stackrel{d f}{=} \iota y(\mathrm{P}(0, y)) \\
& \bar{n} \stackrel{d f}{=} \iota y \exists x_{1} \ldots \exists x_{n-1}\left(\mathrm{P}\left(0, x_{1}\right) \wedge \cdots \wedge \mathrm{P}\left(x_{n-1}, y\right)\right), \text { for } n>1
\end{aligned}
$$

Thus, $\bar{n}$ means something like: the unique thing that is $n$ P-steps downstream from zero. We know from (R0e) and (R0u) that, in each case, the description is proper and, moreover, that $\mathrm{P}(\bar{n}, \overline{n+1})$.

By itself, of course, this does not help: It simply makes it clear where the problem is. But it turns out that the essential undecidability of $R$ does not at all depend upon the availability of (R0e), (R1e), and (R2e), or even of (R0u), (R1u), and (R2u). What we need to know is only, e.g., that each pair of numbers has a unique sum, not that every pair of numbers has a unique sum. That is, we can weaken $R^{*}$ to the theory $R^{\prime}$ that has the following axiom schemes: ${ }^{20}$

$$
\begin{aligned}
& \exists x[\mathrm{P}(\bar{n}, x) \wedge \forall y(\mathrm{P}(\bar{n}, y) \rightarrow x=y)] \\
& \mathrm{A}(\bar{n}, \bar{m}, \overline{n+m}) \wedge \forall x(\mathrm{~A}(\bar{n}, \bar{m}, x) \rightarrow x=\overline{n+m}) \\
& \mathrm{M}(\bar{n}, \bar{m}, \overline{n \times m}) \wedge \forall x(\mathrm{M}(\bar{n}, \bar{m}, x) \rightarrow x=\overline{n \times m}) \\
& \bar{n} \neq \bar{m}, \text { whenever } n \neq m \\
& x \leq \bar{n} \equiv x=\overline{0} \vee \cdots \vee x=\bar{n} \\
& x \leq \bar{n} \vee \bar{n} \leq x
\end{aligned}
$$

Here, $\left(\mathrm{R} 1^{\prime}\right)$ incorportates $\left(\mathrm{R} 1^{*}\right)$ and immediately yields the relevant case of (R1e), and it contains an additional conjunct that gives us the relevant case of $(\mathrm{R} 1 \mathrm{u})$. The same is true for the various replacements for (R2).

There are a couple of different ways to see that $R^{\prime}$ is essentially undecidable. One is simply to reflect upon the proof that every recursive function is representable in $R$ and to note that we nowhere need to know that successors, sums, and products exist and are unique in every case. ${ }^{21}$ It is enough that they should exist and be unique in each case. The other is to note that $R^{\prime}$ interprets $R^{*}$, which trivially interprets $R$. The proof

${ }^{19}$ This is really equivalent to:

$$
\begin{aligned}
\overline{0} & \stackrel{d f}{=} ` \\
\overline{n+1} & \stackrel{d f}{=} \iota y(\mathrm{P}(\bar{n}, y))
\end{aligned}
$$

though unwinding this one takes more work.

${ }^{20}$ Note that $\left(\mathrm{R} 1^{\prime}\right)$ and $\left(\mathrm{R} 2^{\prime}\right)$ say precisely that addition and multiplication are numeralwise representable.

${ }^{21}$ This kind of argument is worked out in detail by Hájek (2007), though he is concerned with the stronger theory $\mathrm{Q}^{h}$ that we shall meet in Section 7. 
does not involve sophisticated techniques. One need only arrange for there to be a (unique) throwaway value in the cases where a unique value does not already exist. One can do whatever one likes with $S$ and $\times$, but some care is needed with addition, due to how $\leq$ is defined. ${ }^{22}$ If we were to let $a+b=0$, if $a+b$ is not already defined, then that would imply that $b \leq 0$, which could invalidate (R4'). But it suffices to let $a+b=b$, since that only implies that $b \leq b$, which will not cause problems. ${ }^{23}$

\section{Interpreting $R_{0}^{\prime}$ in Adjunctive Frege Arithmetic}

Our initial goal, then, is to show that $\mathrm{R}^{\prime}$ can be interpreted in predicative Frege arithmetic. But we can do even better.

It was observed by Alan Cobham (1960) that, if we drop (R5) from R, then the resulting theory $R_{0}$ is still essentially undecidable. ${ }^{24}$ The same is true of the relational version, $\mathrm{R}_{0}^{\prime}$, which has as axioms all instances of $\left(\mathrm{R} 0^{\prime}\right)-\left(\mathrm{R} 4^{\prime}\right){ }^{25}$ And it turns out that $\mathrm{R}_{0}^{\prime}$ can be naturally interpreted in a version of FA whose logic is even weaker than predicative second-order logic. Following Visser (2011), we might call it 'adjunctive logic'. It has the following six axioms: ${ }^{26}$

$$
\begin{aligned}
& \exists F \forall x(\neg F x) \\
& \forall F \forall y \exists G \forall x(G x \equiv F x \vee x=y)
\end{aligned}
$$

$$
\exists R \forall x \forall y(\neg R x y)
$$

$$
\forall R \forall x \forall y \exists Q \forall z \forall w(Q z w \equiv R z w \vee(z=x \wedge w=y))
$$

\footnotetext{
${ }^{22}$ If we treat $\leq$ as primitive, of course, then this problem does not arise, and we can do as we wish with addition, too. One might then simply note that the version of $R^{\prime}$ in which $\leq$ is defined trivially interprets the version in which it is primitive: Just use the definition of $\leq$ as an interpretation of it.

${ }^{23}$ Thanks to Albert Visser for confirming all of this for me.

${ }^{24}$ To interpret $\mathrm{R}_{0}$ in $\mathrm{R}$, simply define:

$$
x \leq^{\prime} y \equiv[0 \leq y \wedge \forall u(u \leq y \wedge u \neq y \rightarrow \mathrm{S} u \leq y) \rightarrow x \leq y]
$$

Then (R4) still holds, and (R5) can be proven (Jones and Shepherdson, 1983, p. 62). This assumes, of course, that $\leq$ is primitive, not defined. But if the version with $\leq$ taken as primitive is essentially undecidable, then so is the version in which it is treated as defined. As noted earlier, the definition amounts to an interpretation.

By the way, Jones and Sheperdson (1983, pp. 62-3) go even further and show that the theory with just the schemes (R2), (R3), and (R4) in the language $\times, \leq$, with the numerals treated as primitive constants, is also essentially undecidable. The argument involves the use of an ingenious definition of addition and successor in terms of multiplication due to Julia Robinson (1949). But that will not help us.

${ }^{25}$ This is because $R_{0}^{\prime}$ interprets $R_{0}$, via the same construction as at the end of the previous section.

${ }^{26}$ The list can be continued in the obvious way, but we shall need only this much.
} 
Adjunctive logic tells us that empty concepts and relations exist, and it allows us to 'adjoin' an additional object to any concept we already have (or a pair or triple, as the case might be, to any relation we already have).

Let $\phi$ be a disjunction of identity-statements: $x=t_{1} \vee \cdots \vee x=t_{n}$. Then (a1e) and $n$ applications of (a1a) yield comprehension for $\phi$ :

$$
\exists F \forall x\left(F x \equiv x=t_{1} \vee \cdots \vee x=t_{n}\right)
$$

Similarly, instances of comprehension of the form

$$
\exists R \forall x \forall y\left[R x y \equiv\left(x=t_{1} \wedge y=u_{1}\right) \vee \cdots \vee\left(x=t_{n} \wedge y=u_{n}\right)\right]
$$

follow from (a2e) and (a2a), and the same holds for three-place relations. Most of the concepts and relations we need to know exist will be of these forms.

Let adjunctive Frege arithmetic (AFA) be adjunctive logic plus HP. Then the claim is that $R_{0}^{\prime}$ can be 'naturally' interpreted in AFA. We have already seen the definitions of zero, predecession, and addition, but not yet the definition of multiplication. The idea behind that defintion is to take $\mathrm{M}(\mathrm{N} x: F x, \mathrm{~N} x: G x, \mathrm{~N} x: H x)$ to be true just in case there is a one-one correspondence between $H$ and the pairs we can form from the $F$ s and the $G$ s. We do not have pairing in AFA, so we cannot do quite that, but we can do something similar.

Think of the three-place relation $R x y z$ as if it were a function from the pair $\langle x, y>$ to $z$. We say that $R$ correlates $F$ and $G$ one-one with $H$ just in case, as such a function, $R$ is one-one

$$
\begin{gathered}
\forall x_{1} \forall y_{1} \forall z_{1} \forall x_{2} \forall y_{2} \forall z_{2}\left[R x_{1} y_{1} z_{1} \wedge R x_{2} y_{2} z_{2} \rightarrow\right. \\
\left.\left(x_{1}=x_{2} \wedge y_{1}=y_{2}\right) \equiv z_{1}=z_{2}\right]
\end{gathered}
$$

and it maps $F \times G$ onto $H$ :

$$
\begin{gathered}
\forall x \forall y[F x \wedge G y \rightarrow \exists z(H z \wedge R x y z)] \wedge \\
\forall z[H z \rightarrow \exists x \exists y(F x \wedge G y \wedge R x y z)]
\end{gathered}
$$

Abbreviate the conjunction of these two statements as: $F \times G \stackrel{R}{\approx} H$. Then we define multiplication as follows:

$\mathrm{M}(a, b, c) \stackrel{d f}{=} \exists F \exists G[a=\mathrm{N} x: F x \wedge b=\mathrm{N} x: G x \wedge \exists H(F \times G \stackrel{R}{\approx} H \wedge c=\mathrm{N} x: H x)]$

The translation on which our interpretation is based is thus the one given in Table 1 on page $12 .{ }^{27}$ There is no need to restrict the domain, so the interpretation we shall in fact be giving is unrelativized.

Showing that these definitions allow us to interpret $R_{0}^{\prime}$ is then straightforward. I shall illustrate by proving representative instances of each of the various schemata. It will be clear enough that the other instances can be proved similarly, but I shall omit the ugly proof that they all can

\footnotetext{
${ }^{27}$ If we had taken $\leq$ as primitive, then we would also need to interpret it. We could do so via its definition in terms of addition, if we wished, but a more 'natural' definition
} 


$$
\begin{aligned}
& 0 \stackrel{d f}{=} \mathrm{N} x: x \neq x \\
& \mathrm{P} a b \stackrel{d f}{=} \exists F \exists y[b=\mathrm{N} x: F x \wedge F y \wedge a=\mathrm{N} x:(F x \wedge x \neq y)] \\
& \mathrm{A}(a, b, c) \stackrel{d f}{=} \exists F \exists G[a=\mathrm{N} x: F x \wedge b=\mathrm{N} x: G x \wedge \\
&\neg \exists x(F x \wedge G x) \wedge c=\mathrm{N} x:(F x \vee G x)] \\
& \mathrm{M}(a, b, c) \stackrel{d f}{=} \exists F \exists G \exists H[a=\mathrm{N} x: F x \wedge b=\mathrm{N} x: G x \wedge c=\mathrm{N} x: H x \wedge \\
&F \times G \stackrel{R}{\approx} H]
\end{aligned}
$$

TABLE 1. The Interpretation

be (which is by induction, of course, in each case). I will do the case of predecession in a little more detail than the others, so we can get a feel for how these proofs go. The rest I shall do a bit more quickly.

\subsection{Some Useful Lemmas.}

Lemma 1. For each formula $A(x)$, AFA proves:

$$
\mathrm{N} x: A(x)=0 \equiv \neg \exists x(A(x))
$$

Proof. Left to right: By HP, some $R$ correlates $A(\xi)$ one-one with $\xi \neq \xi$. Hence, $\forall x(A(x) \rightarrow \exists y(y \neq y \wedge R x y))$, so $\neg \exists x(A(x))$.

Right to left: Let $Q \xi \eta$ be the empty relation. ${ }^{28}$ If $\neg \exists x(A(x))$, then $Q \xi \eta$ correlates $A(\xi)$ one-one with $\xi \neq \xi$. So $\mathrm{N} x: A(x)=\mathrm{N} x:(x \neq x)=0$.

Many of the arguments I am about to give would be easier if we knew that $\mathrm{N} x: \phi x$ was extensional, i.e., that

$$
\forall x(A(x) \equiv B(x)) \rightarrow \mathrm{N} x: A(x)=\mathrm{N} x: B(x)
$$

Boolos (1998d, pp. 279-80) calls this principle 'Log' on the ground that it has a reasonable claim to be a truth of logic. It does indeed, but we do not have it in AFA, there is no hope of our proving it, and it is best to avoid it where we can. So I shall instead prove a meta-theorem that does much of the work of Log.

would be:

$$
\begin{aligned}
a \leq^{*} b \stackrel{d f}{=} \exists F \exists G\{a=\mathrm{N} x: F x \wedge b=\mathrm{N} x: G x \wedge \\
\exists R[\forall x \forall y \forall z \forall w(R x z \wedge R y w \rightarrow x=z \equiv y=w) \wedge \\
\forall x(F x \rightarrow \exists y(G y \wedge R x y)]\}
\end{aligned}
$$

which says that $\mathrm{N} x: F x \leq \mathrm{N} x: G x$ if the $F$ s can be mapped one-one into the $G$ s. We can show that $a \leq b \rightarrow a \leq^{*} b$ in simple FA. To prove the converse, we seem to need predicative comprehension. Nonetheless, this alternative defintion is also adequate for the proof of $\left(\mathrm{R} 4^{\prime}\right)$, as I shall note below.

${ }^{28}$ So we need only (a2e) for this argument. 
Lemma 2. Suppose that $A(x)$ is a disjunction of identities. Let $B(x)$ be any formula. Then AFA proves:

$$
\forall x(A(x) \equiv B(x)) \rightarrow \mathbf{N} x: A(x)=\mathbf{N} x: B(x)
$$

Proof. Consider a simple case. Let $A(\xi)$ be: $\xi=\overline{0} \vee \xi=\overline{1}$. Now let $Q a b$ be: $(a=\overline{0} \wedge b=\overline{0}) \vee(a=\overline{1} \wedge b=\overline{1})$. Then $Q$ exists, and it is easy to show, using the fact that $\forall x(A(x) \equiv B(x))$, that $Q$ correlates $A(\xi)$ one-one with $B(\xi)$.

A similar but more general result can be proven in much the same way.

Lemma 3. For any formulae $A(x)$ and $B(x)$, AFA proves:

$$
\begin{gathered}
\forall x\left(A(x) \equiv x=a_{1} \vee \cdots \vee x=a_{n}\right) \wedge \\
\forall x\left(B(x) \equiv x=b_{1} \vee \cdots \vee x=b_{n}\right) \wedge \\
\bigwedge_{i \neq j}\left(a_{i} \neq a_{j}\right) \wedge \bigwedge_{\substack{i \neq j \\
(}}\left(b_{i} \neq b_{j}\right) \rightarrow \\
\mathrm{N} x: A(x)=\mathrm{N} x: B(x)
\end{gathered}
$$

Proof. Let $Q a b$ be: $\left(a=a_{1} \wedge b=b_{1}\right) \vee \cdots \vee\left(a=a_{n} \wedge b=b_{n}\right)$.

We have a corresponding result for inequality.

Lemma 4. For any formulae $A(x)$ and $B(x)$, if $m \neq n$, AFA proves:

$$
\begin{aligned}
& \forall x\left(A(x) \equiv x=a_{1} \vee \cdots \vee x=a_{m}\right) \wedge \\
& \forall x\left(B(x) \equiv x=b_{1} \vee \cdots \vee x=b_{n}\right) \wedge \\
& \bigwedge_{i \neq j}\left(a_{i} \neq a_{j}\right) \wedge \bigwedge_{i \neq j}\left(b_{i} \neq b_{j}\right) \rightarrow \\
& \mathrm{N} x: A(x) \neq \mathbf{N} x: B(x)
\end{aligned}
$$

Proof. It is a simple exercise in first-order reasoning to show that, under the hypotheses of the theorem, if we assume that $R$ correlates $A(\xi)$ one-one with $B(\xi)$, then we can reach a contradiction.

Lemma 5. For any formula $A(x)$, and for each $n$, AFA proves:

$$
\begin{gathered}
\mathrm{N} x:\left(x=a_{1} \vee \cdots \vee x=a_{n}\right)=\mathrm{N} x: A(x) \wedge \bigwedge_{i \neq j}\left(a_{i} \neq a_{j}\right) \rightarrow \\
\exists y_{1} \cdots \exists y_{n}\left[\bigwedge_{\substack{i \neq j\\
}}\left(y_{i} \neq y_{j}\right) \wedge \forall z\left(A(z) \equiv\left(z=y_{1} \vee \cdots \vee z=y_{n}\right)\right)\right]
\end{gathered}
$$

Proof. Assume the antecedent. Then some $R$ correlates $\xi=a_{1} \vee \cdots \vee \xi=$ $a_{n}$ one-one with $A(\xi)$. The $y_{i}$ are the images of the $a_{i}$ under $R$. Their distinctness follows from the fact that $R$ is one-one. The final clause follows from the fact that $R$ is onto. 
In the remainder of this section, then, we shall show that the instances of $\left(\mathrm{R} 0^{\prime}\right)-\left(\mathrm{R} 4^{\prime}\right)$ can all be proven in AFA. Except in the case of $\left(\mathrm{R} 2^{\prime}\right)$, multiplication, we shall need to know no more about numbers than is contained in the lemmata just proven. This fact is of interest because they can themselves be proven from something much weaker than HP, namely, what I have elsewhere called HPJ (Heck, 2011a, p. 170):

$$
\mathrm{N} x: A(x)=\mathrm{N} x: B(x) \equiv \operatorname{JAM}_{x}(A(x), B(x))
$$

Here, 'JAM $(A(x), B(x))$ ' means: There are just as many $A$ s as $B$ s, and we may take it to be characterized by the following three principles:

$\left(\mathrm{ZCE}^{*}\right) \quad \neg \exists x(A(x)) \rightarrow\left[\mathrm{JAM}_{x}(A(x), B(x)) \equiv \neg \exists x(B(x))\right]$

(APC*) $\quad \operatorname{JAM}_{x}(A(x), B(x)) \wedge \neg A(a) \wedge \neg B(b) \rightarrow$

$$
\mathrm{JAM}_{x}(A(x) \vee x=a, B(x) \vee x=b)
$$

(RPC*) $\quad \operatorname{JAM}_{x}(A(x), B(x)) \wedge A(a) \wedge B(b) \rightarrow$

$$
\operatorname{JAM}_{x}(A(x) \wedge x \neq a, B(x) \wedge x \neq b)
$$

More seems to be needed for ( $\mathrm{R}^{\prime}$ ), however, because the definition of multiplication is somewhat complicated.

4.2. Schemata $\left(\mathbf{R 0}^{\prime}\right)$ and $\left(\mathbf{R 3}^{\prime}\right)$. We now turn our attention to schema $\left(\mathrm{R}^{\prime}\right)$. For reasons that will become clear, we will also establish, as we proceed, the relevant instances of schema $\left(\mathrm{R} 3^{\prime}\right)$.

The first instance of $\left(\mathrm{R}^{\prime}\right)$ is:

$$
\exists x(\mathrm{P}(0, x) \wedge \forall y(\mathrm{P}(0, y) \rightarrow x=y))
$$

We take $x$ to be: $\mathrm{N} x: x=0$. We first need to show that $\mathrm{P}(0, \mathbf{N} x: x=0)$, i.e., that

$$
\exists F \exists y[\mathbf{N} x:(x=0)=\mathbf{N} x: F y \wedge F y \wedge 0=\mathbf{N} x:(x=0 \wedge x \neq y)]
$$

We take $F \xi$ to be: $\xi=0$, which exists by adjunctive comprehension, and $y$ to be: 0 . So we need to prove:

$$
\mathrm{N} x:(x=0)=\mathrm{N} x:(x=0) \wedge 0=0 \wedge 0=\mathrm{N} x:(x=0 \wedge x \neq 0)
$$

This is trivial, except for the last conjunct, and it follows from Lemma 1.

We now need to establish uniqueness. So suppose $\mathrm{P}(0, y)$; we want to show that $y=\mathrm{N} x:(x=0)$. By the definition of $\mathrm{P}$, for some $F$ and $z$, we have: $y=\mathrm{N} x: F x, F z$, and $\mathrm{N} x:(x \neq x)=\mathrm{N} x:(F x \wedge x \neq z)$. By Lemma 1, nothing falls under $F \xi \wedge \xi \neq z$, so $F x \equiv x=z$, whence $\mathrm{N} x: F x=\mathrm{N} x:(x=0)$, by Lemma 3 .

It follows that: $\overline{1}=\mathrm{N} x:(x=0)$; so we might as well abbreviate 'N $x:(x=0)$ ' as: 1 .

We now dispose of an instance of $\left(\mathrm{R}^{\prime}\right)$, namely: $\overline{0} \neq \overline{1}$, that is: $\mathrm{N} x:(x \neq$ $x) \neq \mathrm{N} x:(x=0)$. This follows from Lemma 1 .

The next instance of $\left(\mathrm{R}^{\prime}\right)$ is:

$$
\exists z(\mathrm{P}(\overline{1}, z) \wedge \underset{14}{\forall y(\mathrm{P}(\overline{1}, y) \rightarrow z=y)}
$$


In virtue of what we just did, this is equivalent to:

$$
\exists z(\mathrm{P}(\mathrm{N} x:(x=0), z) \wedge \forall y(\mathrm{P}(\mathrm{N} x:(x=0), y) \rightarrow z=y)
$$

We take $z$ to be: $\mathrm{N} x:(x=0 \vee x=1)$ and first prove that $\mathrm{P}(\mathrm{N} x: x=$ $0, \mathbf{N} x:(x=0 \vee x=1))$, that is:

$$
\begin{gathered}
\exists F \exists y[\mathrm{~N} x:(x=0 \vee x=1)=\mathrm{N} x: F x \wedge F y \wedge \\
\mathbf{N} x:(x=0)=\mathbf{N} x:(F x \wedge x \neq y)]
\end{gathered}
$$

We take $F \xi$ to be: $\xi=0 \vee \xi=1$, and $y$ to be: 1 . So we need to prove:

$$
\begin{gathered}
\mathrm{N} x:(x=0 \vee x=1)=\mathrm{N} x:(x=0 \vee x=1) \wedge(1=0 \vee 1=1) \wedge \\
\mathrm{N} x:(x=0)=\mathrm{N} x:((x=0 \vee x=1) \wedge x \neq 1)
\end{gathered}
$$

The first two conjuncts are again trivial. The last will follow from Lemma 2 if we can show that $\forall x(x=0 \equiv((x=0 \vee x=1) \wedge x \neq 1))$. But that follows from $0 \neq 1$, which is why we needed to collect that instance of (R3').

We now need to establish uniqueness. So suppose $\mathrm{P}(\mathrm{N} x:(x=0), y)$; we need to show that $y=\mathrm{N} x:(x=0 \vee x=1)$. By the definition of $\mathrm{P}$, for some $F$ and $z$, we have: $y=\mathrm{N} x: F x, F z$, and $\mathrm{N} x:(x=0)=\mathrm{N} x:(F x \wedge x \neq z)$. By Lemma 5, there is exactly one thing, $w$, that falls under $F \xi \wedge \xi \neq z$, so $F x \equiv(x=z \vee x=w)$, so $\mathrm{N} x: F x=\mathrm{N} x:(x=0 \vee x=1)$, by Lemma 3 .

It thus follows that: $\overline{2}=\mathrm{N} x:(x=0 \vee x=1)$, so we can abbreviate 'N $x:(x=0 \vee x=1)$ ' as: 2 .

Again, we collect a couple instances of schema $\left(\mathrm{R}^{\prime}\right): \overline{0} \neq \overline{2}$ and $\overline{1} \neq \overline{2}$. These follow from Lemma 4.

The pattern should now be obvious. $n$ will quite generally abbreviate: $\mathrm{N} x:(x=0 \vee \cdots \vee x=n-1)$. Its successor will be: $\mathbf{N} x:(x=0 \vee \cdots \vee x=n)$. We will then need to show that

$$
\mathrm{P}(\mathrm{N} x:(x=0 \vee \cdots \vee x=n-1), \mathbf{N} x:(x=0 \vee \cdots \vee x=n))
$$

that is, that

$$
\begin{gathered}
\exists F \exists y[\mathbf{N} x:(x=0 \vee \cdots \vee x=n)=\mathbf{N} x: F x \wedge F y \wedge \\
\mathbf{N} x:(x=0 \vee \cdots \vee x=n-1)=\mathbf{N} x:(F x \wedge x \neq y)]
\end{gathered}
$$

We take $F \xi$ to be: $\xi=0 \vee \cdots \vee \xi=n$, and $y$ to be: $n$. So we have to prove:

$$
\begin{gathered}
\mathrm{N} x:(x=0 \vee \cdots \vee x=n)=\mathrm{N} x:(x=0 \vee \cdots \vee x=n) \wedge \\
(n=0 \vee \cdots \vee n=n) \wedge \\
\mathrm{N} x:(x=0 \vee \cdots \vee x=n-1)=\mathbf{N} x:((x=0 \vee \cdots \vee x=n) \wedge x \neq y)]
\end{gathered}
$$

Only the last needs proving, and the relevant instances of (R3') will imply that:

$$
\forall x[(x=0 \vee \cdots \vee x=n) \wedge x \neq n) \equiv(x=0 \vee \cdots \vee x=n-1)]
$$

We can then appeal to Lemma 2. 
For uniqueness, the definition of $\mathrm{P}$ gives us $F$ and $z$ such that $y=$ $\mathrm{N} x: F x$ and $F z$ and $\mathrm{N} x:(x=0 \vee \cdots \vee x=n-1)=\mathrm{N} x:(F x \wedge x \neq z)$; we will need to show that $y=\mathrm{N} x:(x=0 \vee \cdots \vee x=n)$. By Lemma 5 , there are $x_{1}, \ldots, x_{n}$, all distinct, that are all and only the things that fall under $F \xi \wedge \xi \neq z$. So $F x \equiv\left(x=x_{1} \vee \cdots \vee x=x_{n} \vee x=z\right)$, and Lemma 3 and previously established instances of (R3') imply that $\mathrm{N} x: F x=\mathrm{N} x:(x=0 \vee \cdots \vee x=n)$. The 'new' instances of $\left(\mathrm{R}^{\prime}\right)$ will then follow from Lemma 4.

So that takes care of schemata $\left(\mathrm{R}^{\prime}\right)$ and $\left(\mathrm{R}^{\prime}\right)$.

4.3. Schema $\left(\mathbf{R 1 ^ { \prime }}\right)$. For schema $\left(\mathrm{R} 1^{\prime}\right)$, let us look at the case of $2+1=3$ :

$$
\mathrm{A}(\overline{2}, \overline{1}, \overline{2+1}) \wedge \forall x(\mathrm{~A}(\overline{2}, \overline{1}, x) \rightarrow x=\overline{2+1})
$$

In light of the preceding, this becomes:

$$
\begin{gathered}
\mathrm{A}(\mathrm{N} x:(x=0 \vee x=1), \mathrm{N} x:(x=0), \mathrm{N} x:(x=0 \vee x=1 \vee x=2)) \wedge \\
\forall y[\mathrm{~A}(\mathrm{~N} x:(x=0 \vee x=1), \mathrm{N} x:(x=0), y) \rightarrow \\
y=\mathrm{N} x:(x=0 \vee x=1 \vee x=2)]
\end{gathered}
$$

We start by proving the first conjunct and so need to find $F$ and $G$ such that: ${ }^{29}$

$$
\begin{aligned}
& \mathbf{N} x: F x=\mathbf{N} x:(x=0 \vee x=1) \\
& \mathbf{N} x: G x=\mathbf{N} x:(x=0) \\
& \neg \exists x(F x \wedge G x) \\
& \mathbf{N} x:(F x \vee G x)=\mathbf{N} x:(x=0 \vee x=1 \vee x=2)
\end{aligned}
$$

Take $F \xi$ to be: $\xi=0 \vee \xi=1$; take $G \xi$ to be $\xi=2$. Then (c) follows from various instances of schema (R3'). And (a), (b), and (d) follow from those same instances and Lemma 3.

The proof of uniqueness is similar. Suppose that we have $F$ and $G$ such that $\mathbf{N} x: F x=\mathrm{N} x:(x=0 \vee x=1), \mathbf{N} x: G x=\mathrm{N} x: x=0, \neg \exists x(F x \wedge G x)$, and $\mathrm{N} x:(F x \vee G x)=z$; we want to show that

$$
z=\mathrm{N} x:(x=0 \vee x=1 \vee x=2)
$$

i.e., that:

$$
\mathrm{N} x:(F x \vee G x) \equiv \mathrm{N} x:(x=0 \vee x=1 \vee x=2)
$$

By Lemma 5, there are $f_{1}$ and $f_{2}$ such that $f_{1} \neq f_{2}$ and $F x \equiv(x=$ $\left.f_{1} \vee x=f_{2}\right)$, and there is a $g_{1}$ such that $G x \equiv\left(x=g_{1}\right)$. So $F x \vee G x \equiv$ $\left(x=f_{1} \vee x=f_{2} \vee x=g_{1}\right)$, and since $\neg \exists x(F x \wedge G x), f_{i} \neq g_{j}$, for all $i, j$. So our conclusion follows from Lemma 3 , yet again.

\footnotetext{
${ }^{29}$ It is here that we find the stumbling block to a general proof of the existence of sums: We cannot show, quite generally, that such disjoint $F$ and $G$ exist. Even if we had induction, we would not have it for $\exists F \exists G(\neg \exists x(F x \wedge G x) \wedge \ldots)$, due to predicativity restrictions.
} 
4.4. Schema (R2'). For schema $\left(\mathrm{R}^{\prime}\right)$, let us look at the case of $2 \times 2=4$ :

$$
\mathrm{M}(\overline{2}, \overline{2}, \overline{2 \times 2}) \wedge \forall x(\mathrm{M}(\overline{2}, \overline{2}, x) \rightarrow x=\overline{2 \times 2})
$$

That is:

$$
\begin{gathered}
\mathrm{M}(\mathrm{N} x:(x=0 \vee x=1), \mathrm{N} x:(x=0 \vee x=1), \\
\mathrm{N} x:(x=0 \vee x=1 \vee x=2 \vee x=3)) \wedge \\
\forall y[\mathrm{M}(\mathrm{N} x:(x=0 \vee x=1), \mathrm{N} x:(x=0 \vee x=1), y) \rightarrow \\
y=\mathrm{N} x:(x=0 \vee x=1 \vee x=2 \vee x=3)]
\end{gathered}
$$

So we need to show that the 'pairs' we can form from $\xi=0 \vee \xi=1$ and $\xi=0 \vee \xi=1$ can be correlated one-one with the objects falling under $\xi=0 \vee \xi=1 \vee \xi=2 \vee \xi=3$. This is simple. Just take Rabc to be:

$$
\begin{aligned}
& (a=0 \wedge b=0 \wedge c=0) \vee(a=0 \wedge b=1 \wedge c=1) \vee \\
& (a=1 \wedge b=0 \wedge c=2) \vee(a=1 \wedge b=1 \wedge c=3)
\end{aligned}
$$

Then it is easy to show that $R$ is one-one, in the relevant sense, and that it correlates $\xi=0 \vee \xi=1$ and $\xi=0 \vee \xi=1$ with $\xi=0 \vee \xi=1 \vee \xi=2 \vee \xi=3$.

For uniqueness, suppose there are $F, G, H$, and $R$ such that $\mathrm{N} x: F x=$ $\mathrm{N} x:(x=0 \vee x=1), \mathbf{N} x: G x=\mathrm{N} x:(x=0 \vee x=1), y=\mathrm{N} x: H x$, and $F \times G \stackrel{R}{\approx} H$. Then there will be $h_{1}, h_{2}, h_{3}, h_{4}$, all distinct, such that $H x \equiv\left(x=h_{1} \vee x=h_{2} \vee x=h_{3} \vee x=h_{4}\right)$. We can then use Lemma 3 to conclude that $\mathrm{N} x: H x=\mathrm{N} x:(x=0 \vee x=1 \vee x=2 \vee x=3)$.

4.5. Schema (R4'). Finally, then, we need to look at schema $\left(\mathrm{R} 4^{\prime}\right)$. We prove the instance:

$$
x \leq \overline{2} \equiv x=\overline{0} \vee x=\overline{1} \vee x=\overline{2}
$$

Recall that $\leq$ is defined as: $x \leq y \stackrel{d f}{=} \exists z(\mathrm{~A}(z, x, y))$.

Right to left: This follows easily from instances of schema (R1'). In particular: $A(\overline{2}, \overline{0}, \overline{2})$, so $\exists z(A(z, \overline{0}, \overline{2})$, whence $\overline{0} \leq \overline{2}$. Similarly, $A(\overline{1}, \overline{1}, \overline{2})$, so $\overline{1} \leq \overline{2}$; and $A(\overline{0}, \overline{2}, \overline{2})$, so $\overline{2} \leq \overline{2} \cdot{ }^{30}$

Left to right: Suppose $n \leq \overline{2}$, i.e., $n \leq \mathrm{N} x:(x=0 \vee x=1)$. So, for some $z, \mathrm{~A}(z, n, \mathrm{~N} x:(x=0 \vee x=1))$. By the definition of addition, for some $F$ and $G$, we have: $z=\mathrm{N} x: F x, n=\mathrm{N} x: G x, \neg \exists x(F x \wedge G x)$, and $\mathrm{N} x:(F x \vee G x)=\mathrm{N} x:(x=0 \vee x=1)$. So, by Lemma 5, there are distinct $a_{1}$ and $a_{2}$ such that $F x \vee G x \equiv\left(x=a_{1} \vee x=a_{2}\right)$. There are then four possibilities:

(i) $\quad G a_{1} \wedge G a_{2}$

By Lemma 3, $\mathrm{N} x: G x=\mathrm{N} x:(x=0 \vee x=1)$, i.e., $\mathrm{N} x: G x=2$, so $n=\overline{2}$.

(ii) $\quad G a_{1} \wedge \neg G a_{2}$

\footnotetext{
${ }^{30}$ If we use the alternative defintion of $\leq$ mentioned in note 27 , then the argument has to be slightly different: Simply consider the different cases and define one-one maps from $\xi \neq \xi, \xi=0$, and $\xi=0 \vee \xi=1$ into $\xi=0 \vee \xi=1$ explicitly.
} 
Then $\mathrm{N} x: G x=1$, so $n=\overline{1}$.

(iii) $\neg G a_{1} \wedge G a_{2}$

Again, $n=\overline{1}$.

(iv) $\neg G a_{1} \wedge \neg G a_{2}$

Then $\mathrm{N} x: G x=0$, so $n=\overline{0}$. So $n=\overline{0} \vee n=\overline{1} \vee n=\overline{2}$, as wanted. ${ }^{31}$

That completes the proof that $R_{0}^{\prime}$ is naturally interpretable in AFA.

In fact, we can do a bit better: It is easy to show that zero has no predecessor. For suppose $\mathrm{P}(n, 0)$. Then, for some $F$ and $y, 0=\mathrm{N} x: F x$, $F y$, and $n=\mathrm{N} x:(F x \wedge x \neq y)$. But, since $F y, 0 \neq \mathrm{N} x: F x$, by Lemma 1 . Contradiction. So $\neg \exists y(\mathrm{P}(y, 0))$.

\section{5. $R_{0}$ Is Naturally Interpretable in Simple FA}

Since predicative second-order logic clearly proves the axioms of adjunctive logic, the foregoing establishes that $\left(\mathrm{R}^{\prime}\right)-\left(\mathrm{R} 4^{\prime}\right)$ are provable in predicative FA, using the 'natural' definitions of $0, P, A$, and $M$ given in Table 1 on page 12. I shall now show that all instances of schema $\left(\mathrm{R}^{\prime}\right)$ are provable in predicative FA and, in fact, are provable in an even weaker theory. In this case, we do need to restrict the domain, since $\left(\mathrm{R}^{\prime}\right)$ clearly assumes that $x$ is a number. ${ }^{32}$ So we restrict the domain to numbers: $\delta(y) \equiv \exists F(y=\mathrm{N} x: F x)$.

It is implicit in the foregoing that AFA proves every instance of the following:

$$
\exists v_{1} \cdots v_{n}\left[\bigwedge_{i \neq j}\left(v_{i} \neq v_{j}\right) \wedge \forall x\left(G x \equiv\left(x=v_{1} \vee \cdots \vee x=v_{n}\right)\right] \rightarrow \mathrm{N} x: G x=\bar{n}\right.
$$

This will prove useful.

We prove a representative instance of $\left(\mathrm{R}^{\prime}\right): n \leq \overline{3} \vee \overline{3} \leq n$. Since $n$ is a number, $n=\mathrm{N} x: F x$, for some $F$. There are four cases to consider:

(i) $\neg \exists x(F x)$

(ii) $\exists x_{1}\left(F x_{1} \wedge \forall y\left(F y \rightarrow x_{1}=y\right)\right)$

(iii) $\exists x_{1} \exists x_{2}\left(F x_{1} \wedge F x_{2} \wedge x_{1} \neq x_{2} \wedge \forall y\left(F y \rightarrow x_{1}=y \vee x_{2}=y\right)\right)$

\footnotetext{
${ }^{31}$ Using the alternative definition of $\leq$ from note 27 make things a little more complicated. Suppose $n \leq \overline{2}$. Then for some $F$ and $G$, where $n=\mathrm{N} x: F x$ and $\overline{2}=\mathrm{N} x: G x$, there is a one-one map from the $F$ s into the $G$ s. The $G$ s are thus $g_{1}$ and $g_{2}$, distinct. Suppose that there were $f_{1}, f_{2}$, and $f_{3}$, all $F \mathbf{s}$, and distinct. Then we will get a contradiction. The rest of the proof is similar to that of $\left(\mathrm{R5}^{\prime}\right)$ below.

${ }^{32}$ Alternatively, one could reformulate $\mathrm{R}_{0}^{\prime}$ itself with a predicate $N x$, saying that $x$ is a number, and then define $N$ accordingly.
} 
In case (i), $\mathrm{N} x: F x=\overline{0}$; in case (ii), $\mathrm{N} x: F x=\overline{1}$; in case (iii), $\mathrm{N} x: F x=\overline{2}$. The relevant instances of ( $\left(\mathrm{R}^{\prime}\right)$ then yield, in each case, that $\mathrm{N} x: F x \leq \overline{3}$, so $n \leq \overline{3}$.

(iv) $\exists x_{1} \exists x_{2} \exists x_{3}\left(x_{1} \neq x_{2} \wedge x_{1} \neq x_{3} \wedge x_{2} \neq x_{3} \wedge F x_{1} \wedge F x_{2} \wedge F x_{3}\right)$

Let $G \xi$ be: $\xi=x_{1} \vee \xi=x_{2} \vee \xi=x_{3}$. Then $\mathrm{N} x: G x=\overline{3}$. Let $H \xi$ be: $F \xi \wedge \xi \neq$ $x_{1} \wedge \xi \neq x_{2} \wedge \xi \neq x_{3}$, which exists by predicative comprehension. I claim that $\mathrm{A}(\mathrm{N} x: H x, \mathrm{~N} x: G x, \mathrm{~N} x: F x)$, whence $\exists z(\mathrm{~A}(z, \mathrm{~N} x: G x, \mathrm{~N} x: F x))$, so $\exists z(\mathrm{~A}(z, \overline{3}, n))$ and therefore $\overline{3} \leq n$. To prove the claim, we need only note that nothing is both $G$ and $H$, which is obvious, and that the relation of identity, which exists by predicative comprehension, correlates $H \xi \vee G \xi$ one-one with $F \xi$, since $\forall x(F x \equiv(H x \vee G x))$.

That completes the proof of schema $\left(\mathrm{R}^{\prime}\right) .^{33}$

The reader will note that predicative comprehension was used at just two points. The first was when we claimed that $F \xi \wedge \xi \neq x_{1} \wedge \xi \neq x_{2} \wedge \xi \neq$ $x_{3}$ exists. This appeal to predicative comprehension could be replaced by an axiom of class subtraction:

$$
\forall F \forall y \exists G \forall x(G x \equiv F x \wedge x \neq y)
$$

The second was when we claimed that the relation of identity exists. This could be replaced by an appeal to the principle Log we met earlier: ${ }^{34}$

$$
\forall x(A(x) \equiv B(x)) \rightarrow \mathrm{N} x: A(x)=\mathrm{N} x: B(x)
$$

So the foregoing shows, as well, that $R_{0}$ is naturally interpretable in AFA plus the subtraction axiom and Log, which I'll call simple FA, or SFA. We shall see that SFA actually proves quite a bit more, and that predicative FA proves a lot more.

\section{More Results}

SFA proves that predecession is one-one, that every number other than zero has a predecessor, and that addition is commutative:

(i) $\mathrm{P}(a, b) \wedge \mathrm{P}(a, c) \rightarrow b=c$

(ii) $\mathrm{P}(a, c) \wedge \mathrm{P}(b, c) \rightarrow a=b$

(iii) $a \neq 0 \rightarrow \exists y(\mathrm{P}(y, a))$

(iv) $\mathrm{A}(a, b, c) \rightarrow \mathrm{A}(b, a, c)$

We assume that SFA has substraction axioms for relations, as well as for concepts.

\footnotetext{
${ }^{33}$ Essentially the same proof works for the alternative definition of $\leq$ mentioned in note 27 .

${ }^{34}$ Alternatively one could prove Log from the assumption that the identity relation exists: $\exists R \forall x \forall y(R x y \equiv x=y)$.
} 
Frege's own proofs of the first three actually use no more than is available in SFA. We prove (i) and leave the proofs of (ii) and (iii) to the reader.

Assume the antecedent of (i). Then we have $F$ and $v$ such that $b=$ $\mathrm{N} x: F x, F v$, and $a=\mathrm{N} x:(F x \wedge x \neq v)$, and we have $G$ and $u$ such that $c=\mathrm{N} x: G x, G u$, and $a=\mathrm{N} x:(G x \wedge x \neq u)$. So $\mathrm{N} x:(F x \wedge x \neq v)=$ $\mathrm{N} x:(G x \wedge x \neq u)$, and so, by HP, there is a relation $R$ that correlates $F \xi \wedge \xi \neq v$ one-one with $G \xi \wedge \xi \neq u$. If we knew that neither $v$ nor $u$ was in the field of $R$, then we could simply define $Q x y$ as: $R x y \vee(x=v \wedge y=u)$, and it would then be easy to show that $Q$ correlates $F \xi$ one-one with $G \xi$. We do not know that, but we can arrange for it. What we really want is: $(R x y \wedge x \neq v \wedge y \neq u) \vee(x=v \wedge y=u)$. And SFA knows that this relation exists. $^{35}$

For (iv), suppose $\mathrm{A}(a, b, c)$. Then there are $H$ and $I$ such that:

$$
a=\mathrm{N} x: H x \wedge b=\mathrm{N} x: I x \wedge \neg \exists x(H x \wedge I x) \wedge c=\mathrm{N} x:(H x \vee I x)
$$

We need to show that $\mathrm{A}(b, a, c)$, that is, that:

$$
\exists F \exists G[b=\mathrm{N} x: F x \wedge a=\mathrm{N} x: G x \wedge \neg \exists x(F x \wedge G x) \wedge c=\mathrm{N} x:(F x \vee G x)]
$$

But if let $F$ be $I$, and $G$ be $H$, what we need to prove is just:

$$
b=\mathrm{N} x: I x \wedge a=\mathrm{N} x: H x \wedge \neg \exists x(I x \wedge H x) \wedge c=\mathrm{N} x:(I x \vee H x)
$$

All we really need to know, therefore, is that $\mathrm{N} x:(H x \vee I x)=\mathrm{N} x:(I x \vee$ $H x)$. This follows from Log.

Suppose we now enrich SFA with comprehension for quantifier-free formulae. Call this Boolean FA, or BFA. Then we can prove that multiplication too is commutative and establish the uniqueness of sums:

(v) $\mathrm{M}(a, b, c) \rightarrow \mathrm{M}(b, a, c)$

(vi) $\mathrm{A}(a, b, c) \wedge \mathrm{A}(a, b, d) \rightarrow c=d$

For (v), the work is simply showing that, if $F \times G \stackrel{R}{\approx} H$, then, for some $Q, G \times F \stackrel{Q}{\approx} H$. But we can define $Q x y z$ as: $R y x z$. That is, the relevant instance of comprehension is: $\exists Q \forall x \forall y \forall z[Q x y z \equiv R y x z]$.

For (vi), suppose the antecedent. Then we have $F_{1}$ and $G_{1}$ such that $a=\mathrm{N} x: F_{1} x, b=\mathrm{N} x: G_{1} x, \neg \exists x\left(F_{1} x \wedge G_{1} x\right)$, and $c=\mathrm{N} x:\left(F_{1} x \vee G_{1} x\right)$. Also, we have $F_{2}$ and $G_{2}$ such that $a=\mathrm{N} x: F_{2} x, b=\mathrm{N} x: G_{2} x, \neg \exists x\left(F_{2} x \wedge G_{2} x\right)$, and $d=\mathrm{N} x:\left(F_{2} x \vee G_{2} x\right)$. So there is a relation $R$ that correlates the $F_{1} \mathrm{~s}$ one-one with the $F_{2} \mathrm{~s}$, and another relation $Q$ that correlates the $G_{1} \mathrm{~S}$

\footnotetext{
${ }^{35}$ It would apear that we could avoid appeal to the subtraction axiom, and so carry out the proof in AFA, if we reformulated HP so that the required relation $R$ had only $F$ s in its domain and $G$ s in its range. (Frege proves this strengthened form of $\mathrm{HP}$ as Theorem 453 of Grundgesetze.) I do not think that would cause any problems elsewhere, but one would have to check.
} 
one-one with the $G_{2} \mathrm{~s}$. The problem now is to 'paste' these together to get a one-one correlation between $F_{1} \xi \vee G_{1} \xi$ and $F_{2} \xi \vee G_{2} \xi$. Define $S x y$ as:

$$
\left(R x y \wedge F_{1} x \wedge F_{2} y\right) \vee\left(Q x y \wedge G_{1} x \wedge G_{2} y\right)
$$

The initial conjuncts are needed to ensure that $S$ will be one-one. It clearly works as advertised.

If, now, we really do make serious use of predicative comprehensionmeaning: for formulae containing only first-order quantifiers-then we can prove the uniqueness of products and the associativity of addition:

(vii) $\mathrm{M}(a, b, c) \wedge \mathrm{M}(a, b, d) \rightarrow c=d$

(viii) $\mathrm{A}(a, b, u) \wedge \mathrm{A}(b, c, v) \rightarrow \mathrm{A}(u, c, z) \equiv \mathrm{A}(a, v, z)^{36}$

For (vii), $\mathrm{M}(a, b, c)$ gives us $F_{1}, G_{1}$, and $H_{1}$ such that $a=\mathrm{N} x: F_{1} x, b=$ $\mathrm{N} x: G_{1} x$, and $c=\mathrm{N} x: H_{1} x$, and a relation $R_{1}$ such that $F_{1} \times G_{1} \stackrel{R_{1}}{\approx} H_{1}$; similarly, $\mathrm{M}(a, b, d)$ gives us $F_{2}, G_{2}$, and $H_{2}$ such that $a=\mathrm{N} x: F_{2} x, b=$ $\mathrm{N} x: G_{2} x$, and $d=\mathrm{N} x: H_{2} x$, and a relation $R_{2}$ such that $F_{2} \times G_{2} \stackrel{R_{2}}{\approx} H_{2}$. So $\mathrm{N} x: F_{1} x=\mathrm{N} x: F_{2} x$, and $\mathrm{N} x: G_{1} x=\mathrm{N} x: G_{2} x$, and there is a $Q_{1}$ that correlates $F_{1} \xi$ one-one with $F_{2} \xi$, and a $Q_{2}$ that does the same for $G_{1} \xi$ and $G_{2} \xi$.

We now need to paste all these together to get a one-one correlation between $H_{1} \xi$ and $H_{2} \xi$. The following diagram illustrates how we can do this:

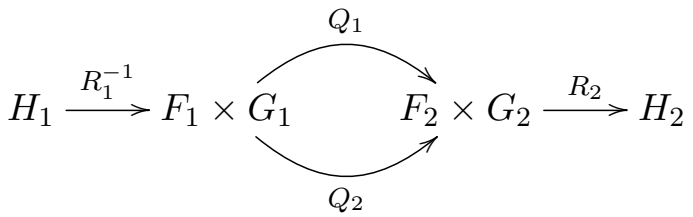

So we define $S a b$ as: ${ }^{37}$

$$
\exists x \exists y\left[R_{1} x y a \wedge \exists z \exists w\left(Q_{1} x z \wedge Q_{2} y w \wedge R_{2} z w b\right)\right]
$$

Predicative comprehension assures us of the existence of $S$, and it is easy, but mind-numbingly tedious, to verify that it correlates $H_{1} \xi$ one-one with $\mathrm{H}_{2} \xi$.

For (viii), assume the antecedent. We prove the left-to-right direction. (The other direction is parallel.) So assume $\mathrm{A}(u, c, z)$. Then we have $F$ and $G$ such that $u=\mathrm{N} x: F x, c=\mathrm{N} x: G x, \neg \exists x(F x \wedge G x)$, and $z=\mathrm{N} x:(F x \vee G x)$. Since $\mathrm{A}(a, b, u)$, we have $H$ and $I$ such that $a=\mathrm{N} x: H x, b=\mathrm{N} x: I x, \neg \exists x(H x \wedge I x)$, and $u=\mathrm{N} x:(H x \vee I x)$. So $\mathrm{N} x: F x=\mathrm{N} x:(H x \vee I x)$. This last fact allows us to 'split' $F$ into two parts: A part corresponding to $H$ and a part corresponding to $I$. More

\footnotetext{
${ }^{36}$ Here, $u=a+b$ and $v=b+c$, so $u+c=(a+b)+c$, and $a+v=a+(b+c)$, so the conclusion asserts that $(a+b)+c=a+(b+c)$.

${ }^{37}$ In effect, the relation $Q_{1} \xi \eta \wedge Q_{2} \zeta v$ is correlating $F_{1} \times G_{1}$ one-one with $F_{2} \times G_{2}$, so we have a chain of equinumerosities.
} 
precisely, HP gives us a relation $R$ that correlates $F \xi$ with $H \xi \vee I \xi$. Define $F_{H} x$ as: $F x \wedge \exists y(R x y \wedge H y)$ and $F_{I} x$ as: $F x \wedge \exists y(R x y \wedge I y)$. These exist by predicative comprehension. It's clear that $\neg \exists x\left(F_{H} x \wedge F_{I} x\right)$ and also that $R$ correlates $F_{H} \xi$ one-one with $H \xi$ and $F_{I} \xi$ one-one with $I \xi$. So, by $\mathrm{HP}, \mathrm{N} x: H x=\mathrm{N} x: F_{H} x$ and $\mathrm{N} x: I x=\mathrm{N} x: F_{I} x$.

We are trying to show that $\mathrm{A}(a, v, z)$. To do so, we need to find $J$ and $K$ such that: $a=\mathrm{N} x: J x, v=\mathrm{N} x: K x, \neg \exists x(J x \wedge K x)$, and $z=\mathrm{N} x:(J x \vee K x)$. We can take $J$ just to be $F_{H}$. We define $K x$ as: $F_{I} x \vee G x$. Since $\neg \exists x(F x \wedge$ $G x)$ and $\neg \exists x\left(F_{I} x \wedge F_{H} x\right), \neg \exists x(J x \wedge K x)$. Moreover, since $\neg \exists x\left(F_{I} x \wedge G x\right)$, $\mathrm{A}\left(\mathbf{N} x: F_{I} x, \mathbf{N} x: G x, \mathbf{N} x:\left(F_{I} x \vee G x\right)\right)$, trivially. Since $\mathrm{A}(b, c, v), b=\mathbf{N} x: F_{I} x$, and $c=\mathrm{N} x: G x$, we have $v=\mathrm{N} x:\left(F_{I} x \vee G x\right)$, by the uniqueness of sums, so $v=\mathbf{N} x: K x$. Finally:

$$
J x \vee K x \equiv F_{I} x \vee\left(F_{H} x \vee G x\right) \equiv F x \vee G x
$$

So, by Log, $\mathrm{N} x:(J x \vee K x)=\mathrm{N} x:(F x \vee G x)=z$, and we are done.

The associativity of multiplication can be proven in a similar way, though the argument is much more complicated. We can do even better: distribution laws, such as $a \times(b+c)=a \times b+a \times c$ can also be proven. The details are ugly_even the properly relational formulations of such claims are ugly_-so I will spare the reader the details. But it is easy enough to convince oneself of these claims by drawing pictures and noting that we just need to rearrange one-one correlations for the proofs. Such arguments just 'feel' predicative.

\section{Relational Versions of $Q$}

We have thus shown that predicative FA naturally interprets relational versions of $R$ and even somewhat stronger theories. Might something similar be done for Q $?^{38}$ It too can be formulated in a purely relational language, and one can then ask the same sorts of questions about it that we have asked about relational versions of $R$.

As it happens, a question in this vicinity was posed a few years ago by Andrzej Grzegorczyk, who asked whether $Q$ remains essentially undecidable if we do not assume that addition and multiplication are total. That is, Grzegorczyk asked whether the theory $\mathrm{Q}^{-}$, which has the following axioms:

$$
\begin{aligned}
& \forall x(\mathrm{~S} x \neq 0) \\
& \forall x \forall y(\mathrm{~S} x=\mathrm{S} y \rightarrow x=y) \\
& \forall x(x \neq 0 \rightarrow \exists y(x=\mathrm{S} y) \\
& \forall x \forall y \forall z \forall w(\mathrm{~A}(x, y, z) \wedge \mathrm{A}(x, y, w) \rightarrow z=w)
\end{aligned}
$$

\footnotetext{
${ }^{38}$ Amazingly, this question failed to occur to me until it was posed by an anonymous referee, who also pointed me in the direction of the relevant literature. What a helpful report!
} 


$$
\begin{aligned}
& \forall x(\mathrm{~A}(x, 0, x)) \\
& \forall x \forall y \forall z[\exists u(\mathrm{~A}(x, y, u) \wedge z=\mathrm{S} u) \rightarrow \mathrm{A}(x, \mathrm{~S} y, z)] \\
& \forall x \forall y \forall z \forall w(\mathrm{M}(x, y, z) \wedge \mathrm{M}(x, y, w) \rightarrow z=w) \\
& \forall x(\mathrm{M}(x, 0,0)) \\
& \forall x \forall y \forall z[\exists u(\mathrm{M}(x, y, u) \wedge \mathrm{A}(u, x, z)) \rightarrow \mathrm{M}(x, \mathrm{~S} y, z)]
\end{aligned}
$$

is essentially undecidable.

Hájek (2007) answered a version of this question, though he considered instead a slightly stronger theory $\mathrm{Q}^{h}$ in which (Q5) and (Q7) are strengthened to biconditionals:

$$
\begin{aligned}
& \forall x \forall y \forall z[\exists u(\mathrm{~A}(x, y, u) \wedge z=\mathrm{S} u) \equiv \mathrm{A}(x, \mathrm{~S} y, z)] \\
& \forall x \forall y \forall z[\exists u(\mathrm{M}(x, y, u) \wedge \mathrm{A}(u, x, z)) \equiv \mathrm{M}(x, \mathrm{~S} y, z)]
\end{aligned}
$$

Hájek carefully analyzed the proof that $Q$ is essentially undecidable and showed that the axioms of $\mathrm{Q}^{h}$ suffice. ${ }^{39}$ Švejdar (2007) then answered Grzegorczyk's original question by showing that $Q$ is interpretable in $Q^{-}$ (and so, of course, in $\mathrm{Q}^{h}$ ).

By itself, of course, this does not help us very much. Successor is still assumed to be total in $\mathrm{Q}^{-}$. As mentioned previously, however, we can prove in ramified FA that every natural number has a successor, where 'natural number' is defined as Frege would have us define it (Heck, 2011e). And this fact can be used, along with what we shall do shortly, to show that $Q^{-}$and $Q^{h}$ can be naturally interpreted in ramified $F A$. But ramified predicative second-order logic is itself somewhat unnatural, so it is worth considering whether we might do without the totality of succession.

The theories in which we will be interested here thus replace (Q1)-(Q3) with the following:

$$
\begin{aligned}
& \mathrm{P}(\bar{n}, \overline{n+1})^{40} \\
& \forall x \forall y \forall z(\mathrm{P}(x, y) \wedge \mathrm{P}(x, z) \rightarrow y=z) \\
& \neg \exists x(\mathrm{P}(x, 0)) \\
& \forall x \forall y \forall z(\mathrm{P}(x, z) \wedge \mathrm{P}(y, z) \rightarrow x=y) \\
& \forall x(x \neq 0 \rightarrow \exists y(\mathrm{P}(y, x)))
\end{aligned}
$$

${ }^{39}$ Hájek also showed that the axioms of a certain fuzzy logic suffice.

${ }^{40}$ Another option here is to use the schema:

$$
\exists x_{1} \cdots \exists x_{n}\left(P 0 x_{1} \wedge \bigwedge_{0<i<n} P x_{i} x_{i+1}\right)
$$

But that is equivalent to what is in the text, given how the numerals are defined. 
Numerals are again defined contextually. We also need to reformulate (Q5) and (Q7) as:

$$
\begin{aligned}
& \forall x \forall y \forall z \forall w[\mathrm{P}(y, w) \wedge \exists u(\mathrm{~A}(x, y, u) \wedge \mathrm{P}(u, z)) \rightarrow \mathrm{A}(x, w, z)] \\
& \forall x \forall y \forall z \forall w[\mathrm{P}(y, w) \wedge \exists u(\mathrm{M}(x, y, u) \wedge \mathrm{A}(u, x, z)) \rightarrow \mathrm{M}(x, w, z)]
\end{aligned}
$$

and similarly for $\left(\mathrm{Q} 5^{h}\right)$ and $\left(\mathrm{Q} 7^{h}\right)$ :

$$
\begin{aligned}
& \forall x \forall y \forall z \forall w[\mathrm{P}(y, w) \rightarrow \exists u(\mathrm{~A}(x, y, u) \wedge \mathrm{P}(u, z)) \equiv \mathrm{A}(x, w, z)] \\
& \forall x \forall y \forall z \forall w[\mathrm{P}(y, w) \rightarrow \exists u(\mathrm{M}(x, y, u) \wedge \mathrm{A}(u, x, z)) \equiv \mathrm{M}(x, w, z)]
\end{aligned}
$$

Call the resulting theories $Q_{R}^{-}$and $Q_{R}^{h}$, respectively. Obviously, these theories are not finitely axiomatized, due to the fact that $(\mathrm{Pe})$ is a schema, so they lack some of the technical interest of $Q$ and $Q^{-}$. But our interest here is not primarily technical.

7.1. The Essential Undecidability of $\mathbf{Q}_{R}^{h}$. I am confident that $Q_{R}^{-}$is essentially undecidable. But a proof that it is appears to involve either using sophisticated techniques such as shortening of cuts or reconstructing, as Hájek does, the proof that every recursive function is representable. By contrast, we can show that $Q_{R}^{h}$ is essentially undecidable in a very straightforward way, namely, by showing that it contains $\mathrm{R}^{\prime}$, in the usual sense that every axiom of $\mathrm{R}^{\prime}$ is a theorem of $\mathrm{Q}_{R}^{h}$. The argument is just an adaptation of the one given by Tarski, Mostowski, and Robinson (1953, pp. 53-4) to show that $Q$ contains $R$.

Recall the schemes that characterize $R^{\prime}$ :

$$
\begin{aligned}
& \exists x[\mathrm{P}(\bar{n}, x) \wedge \forall y(\mathrm{P}(\bar{n}, y) \rightarrow x=y)] \\
& \mathrm{A}(\bar{n}, \bar{m}, \overline{n+m}) \wedge \forall x(\mathrm{~A}(\bar{n}, \bar{m}, x) \rightarrow x=\overline{n+m}) \\
& \mathrm{M}(\bar{n}, \bar{m}, \overline{n \times m}) \wedge \forall x(\mathrm{M}(\bar{n}, \bar{m}, x) \rightarrow x=\overline{n \times m}) \\
& \bar{n} \neq \bar{m}, \text { whenever } n \neq m \\
& x \leq \bar{n} \equiv x=\overline{0} \vee \cdots \vee x=\overline{n-1}
\end{aligned}
$$

The instances of $\left(\mathrm{R}^{\prime}\right)$ are an immediate consequence of $(\mathrm{Pe})$ and $(\mathrm{Pu})$. For $\left(\mathrm{R} 1^{\prime}\right)$, we need only establish the first conjunct of each instance, since the second will then follow from axiom (A) of $Q_{R}^{h}$. But we know that $\mathrm{A}(\bar{n}, \overline{0}, \bar{n})$, by $\left(\mathrm{Q}^{\prime}\right)$. And the following is an instance of $\left(\mathrm{Q}^{\prime}\right)$ :

$$
\forall z[\mathrm{P}(\overline{0}, \overline{1}) \rightarrow \exists u(\mathrm{~A}(\bar{n}, \overline{0}, u) \wedge \mathrm{P}(u, z)) \equiv \mathrm{A}(\bar{n}, \overline{1}, z)]
$$

which reduces to:

$$
\forall z[\exists u(\mathrm{~A}(\bar{n}, \overline{0}, u) \wedge \mathrm{P}(u, z))] \equiv \mathrm{A}(\bar{n}, \overline{1}, z)]
$$

since $\mathrm{P}(\overline{0}, \overline{1})$. But certainly $\mathrm{A}(\bar{n}, \overline{0}, \bar{n}) \wedge \mathrm{P}(\bar{n}, \overline{n+1})$, so $\mathrm{A}(\bar{n}, \overline{1}, \overline{n+1})$. Repeating the argument shows that $\mathrm{A}(\bar{n}, \overline{2}, \overline{n+2})$, and so forth. 
The same sort of reasoning establishes the various instances of $\left(\mathrm{R} 2^{\prime}\right)$. We know that $\mathrm{M}(\bar{n}, \overline{0}, \overline{0})$, by $\left(\mathrm{Q} 6^{\prime}\right)$. By $\left(\mathrm{Q}^{\prime}\right)$,

$$
\forall z[\mathrm{P}(\overline{0}, \overline{1}) \rightarrow \exists u(\mathrm{M}(\bar{n}, \overline{0}, u) \wedge \mathrm{A}(u, \bar{n}, z)) \equiv \mathrm{M}(\bar{n}, \overline{1}, z)]
$$

and so

$$
\forall z[\exists u(\mathrm{M}(\bar{n}, \overline{0}, u) \wedge \mathrm{A}(u, \bar{n}, z)) \equiv \mathrm{M}(\bar{n}, \overline{1}, z)]
$$

But we have that $\mathrm{M}(\bar{n}, \overline{0}, \overline{0}) \wedge \mathrm{A}(\overline{0}, \bar{n}, \overline{n \times 1})$, by $\left(\mathrm{Q} 6^{\prime}\right)$ and $\left(\mathrm{R} 1^{\prime}\right)$, whence $\mathrm{M}(\bar{n}, \overline{1}, \overline{n \times 1})$. Repeating the argument shows that $\mathrm{M}(\bar{n}, \overline{2}, \overline{n \times 2})$, and so forth.

For $\left(\mathrm{R}^{\prime}\right)$, that $\overline{0} \neq \overline{1}$ follows from $\mathrm{P}(\overline{0}, \overline{1})$ and $\left(\mathrm{Q} 1^{\prime}\right)$. Since $\mathrm{P}(\overline{1}, \overline{2})$, we have: $\exists x \exists y(\mathrm{P}(x, y) \wedge \mathrm{P}(y, \overline{2}))$. But $\neg \exists x \exists y(\mathrm{P}(x, y) \wedge \mathrm{P}(y, \overline{1}))$, since if $\mathrm{P}(y, \overline{1})$, then $y=0$, by $\left(\mathrm{Q} 2^{\prime}\right)$. And so forth.

For $\left(\mathrm{R} 4^{\prime}\right)$, consider first:

$$
x \leq \overline{0} \equiv x=\overline{0}
$$

The right-to-left direction follows from an instance of $\left(\mathrm{R} 1^{\prime}\right)$. So suppose $\exists z(\mathrm{~A}(z, x, \overline{0}))$, and suppose $x \neq 0$. The following is an instance of $\left(\mathrm{Q}^{\prime}\right)$ :

$$
\mathrm{P}(y, x) \rightarrow \exists u(\mathrm{~A}(z, y, u) \wedge \mathrm{P}(u, \overline{0})) \equiv \mathrm{A}(z, x, \overline{0})
$$

Since $x \neq 0, \mathrm{P}(y, x)$, for some $y$, by (Q3'), so we must have:

$$
\exists u(\mathrm{~A}(z, y, u) \wedge \mathrm{P}(u, \overline{0}))
$$

for that same $y$. But that contradicts $\left(\mathrm{Q} 1^{\prime}\right)$.

So suppose

$$
x \leq \bar{n} \equiv x=\overline{0} \vee \cdots \vee x=\bar{n}
$$

We want to show

$$
x \leq \overline{n+1} \equiv x=\overline{0} \vee \cdots \vee x=\overline{n+1}
$$

Again, the right-to-left direction follows from instances of $\left(R 1^{\prime}\right)$. So suppose $\exists z(\mathrm{~A}(z, x, \overline{n+1}))$, and suppose $x \neq 0$. The following is an instance of $\left(\mathrm{Q} 5^{\prime}\right): \mathrm{s}$

$$
\mathrm{P}(y, x) \rightarrow \exists u(\mathrm{~A}(z, y, u) \wedge \mathrm{P}(u, \overline{n+1})) \equiv \mathrm{A}(z, x, \overline{n+1})
$$

Since $x \neq 0, \mathrm{P}(y, x)$, for some $y$, by $\left(\mathrm{Q} 3^{\prime}\right)$ again, so we must have:

$$
\exists u(\mathrm{~A}(z, y, u) \wedge \mathrm{P}(u, \overline{n+1}))
$$

for that same $y$. So $u=\bar{n}$, by $(\mathrm{Pe})$ and $(\mathrm{Pu})$, and so $y \leq \bar{n}$. Hence, by the induction hypothesis, $y=\overline{0} \vee \cdots \vee y=\bar{n}$, so $x=\overline{1} \vee \cdots \vee x=\overline{n+1}$.

$\mathrm{Q}_{R}^{h}$ thus contains $\mathrm{R}^{\prime}$ and so is essentially undecidable. Moreover, it follows immediately that $Q^{h}$ is essentially undecidable, since it obviously interprets $\mathrm{Q}_{R}^{h}$ : Simply define $\mathrm{P}(x, y)$ as: $y=\mathrm{S} x$. This appears to be an improvement over existing proofs.

Note, however, that we really did need the stronger axiom $\left(\mathrm{Q} 5^{\prime}\right)$ of $\mathrm{Q}_{R}^{h}$ rather than the weaker axiom $\left(\mathrm{Q}^{*}\right)$ of $\mathrm{Q}_{R}^{-}$at $(\mathrm{H} 1)$ and $(\mathrm{H} 2)$ above, since we needed to go right to left, not left to right. So this argument does not show that the weaker $Q_{R}^{-}$contains $R^{\prime}$. And, indeed, it is easy to see that 
the axioms of $Q_{R}^{-}$do not preclude there being a non-standard number $b$ for which $\mathrm{A}(b, b, 0)$, in which case not even $x \leq 0 \equiv x=0$ will hold.

7.2. $\mathbf{Q}_{R}^{h}$ Is Naturally Interpretable in Predicative FA. We have already seen that most of the axioms of $Q_{R}^{h}$ are provable in predicative FA. At the end of Section 4, for example, we saw that ( $\left.\mathrm{Q} 1^{\prime}\right)$ is provable in AFA. In Section 6, we saw that $(\mathrm{Pe}),(\mathrm{Pu}),\left(\mathrm{Q} 2^{\prime}\right)$, and $\left(\mathrm{Q}^{\prime}\right)$ are all provable in SFA; that axiom (A) is provable in BFA; and that axiom $(\mathrm{M})$ is provable in predicative FA. So we need only discuss $\left(\mathrm{Q} 4^{\prime}\right)-\left(\mathrm{Q} 7^{\prime}\right)$.

Axiom $\left(\mathrm{Q}^{\prime}\right)$ is provable in SFA. $\mathrm{A}(a, 0, a)$ amounts to:

$$
\exists F \exists G[a=\mathrm{N} x: F x \wedge 0=\mathrm{N} x: G x \wedge \neg \exists x(F x \wedge G x) \wedge a=\mathrm{N} x:(F x \vee G x)]
$$

But we are restricting attention to numbers, so $a=\mathrm{N} x: F x$, for some $F$. Moreover, $0=\mathrm{N} x: x \neq x$ so, by comprehension, $0=\mathrm{N} x: G x$, where $\neg \exists x(G x)$. So $\forall x(F x \vee G x \equiv F x)$, by logic, and then $\mathrm{N} x:(F x \vee G x)=$ $\mathrm{N} x: F x$, by Log. So $a=\mathrm{N} x:(F x \vee G x)$.

Though axiom $\left(\mathrm{Q5}^{\prime}\right)$ can be proven directly, it is enough for our purposes to observe that it is a consequence of associativity, since $a+\mathrm{S} b=$ $\mathrm{S}(a+b)$ is just $a+(b+1)=(a+b)+1$ in disguise. Since the associativity of addition is provable in predicative FA, we need only show that $\mathrm{P}(a, b)$ iff $\mathrm{A}(a, \overline{1}, b)$, which is easy enough to do.

Axiom $\left(\mathrm{Q}^{\prime}\right)$ is provable in AFA. $\mathrm{M}(a, 0,0)$ amounts to:

$$
\exists F \exists G \exists H[a=\mathrm{N} x: F x \wedge 0=\mathrm{N} x: G x \wedge 0=\mathrm{N} x: H x \wedge F \times G \stackrel{R}{\approx} H]
$$

Since both $G$ and $H$ must be empty, what we need to show is that there is a 1-1 relation $R$ such that:

$$
\begin{gathered}
\forall x \forall y[F x \wedge y \neq y \rightarrow \exists z(z \neq z \wedge R x y z)] \wedge \\
\forall z[z \neq z \rightarrow \exists x \exists y(F x \wedge y \neq y \wedge R x y z)]
\end{gathered}
$$

But of course both conjuncts are trivial, and any one-one relation will do. We can thus just let $R$ be the empty relation.

Like ( $\left.\mathrm{Q} 5^{\prime}\right)$, axiom $\left(\mathrm{Q} 7^{\prime}\right)$ can be proven directly, but is also a consequence of the distributivity of multiplication over addition, since $a \times \mathrm{S} b=a \times b+a$ is just $a \times(b+1)=(a \times b)+(a \times 1)$ in disguise. The distribution rules are provable in predicative $\mathrm{FA}$, so we need only show that $\mathrm{M}(a, \overline{1}, a)$, which is trivial.

\section{Summary of the Results}

We have discussed four different theories, which, in order of increasing strength, are:

- Adjunctive FA: HP plus axioms stating the existence of empty concepts and relations and allowing the adjunction of single elements to arbitrary concepts and relations. 
- Simple FA: Adjunctive FA plus (i) axioms allowing the removal of single elements from concepts and relations and (ii) Boolos's axiom Log, stating the extensionality of $\mathrm{N} x: \phi x$.

- Boolean FA: HP plus comprehension for quantifier-free formulae.

- Predicative FA: HP plus predicative comprehension.

We have seen that all of these theories 'naturally' interpret essentially undecidable arithmetical theories. Using the standard definitions of zero, predecession, addition, and multiplication summarized in Table 1 on page 12:

- Adjunctive FA proves the axioms of $\mathrm{R}_{0}^{\prime}$, plus the claim that zero has no predecessor.

- Simple FA proves the axioms of $\mathrm{R}^{\prime}$ and, moreover, that predecession is one-one and that addition is commutative.

- Boolean FA proves further that multiplication is commutative and that sums are unique (when they exist).

- Predicative FA proves yet further that products are unique (when they exist) and that addition and multiplication are associative, and also proves the distribution rules. Moreover, predicative FA proves the axioms of $Q_{R}^{h}$.

Significant chunks of arithmetic are thus derivable from HP using weak fragments of second-order logic.

In the first two cases, these are very weak fragments of second-order logic. What adjunctive logic adds to first-order logic is only the simplest sort of reasoning about sets (or pluralities, or concepts, or what have you). The natural model for adjunctive logic takes the second-order domain to comprise only those sets that can be explicitly specified by listing their elements. Reasoning with such sets seems well within the abilities of ordinary folk, and even of children. And simple FA does not add much more. It has, in fact, precisely the same natural model.

There is a clear technical sense, too, in which these are weak logics.

Proposition. Let $\mathcal{T} \supseteq \mathrm{I} \Delta_{0} .{ }^{41}$ Then $\mathcal{T}$ plus Boolean second-order logic is locally interpretable in $\mathcal{T}$.

Proof. In $\mathrm{I} \Delta_{0}$, we have a partial truth definition for $\Delta_{0}$ formulae, and we can use it to code $\Delta_{0}$ definable sets as (the codes of) $\Delta_{0}$ formulae (Hájek and Pudlák, 1993, pp. 361-65). We can then interpret Boolean second-order quantification as quantification over such sets.

\footnotetext{
${ }^{41} \mathrm{I} \Delta_{0}$ is $\mathrm{Q}$ plus induction for $\Delta_{0}$ formulae, that is, for formulae that contain only 'bounded' quantifiers of the forms $\forall x<t$ and $\exists x<t$.
} 
Nothing of the sort is true for predicative second-order logic, ${ }^{42}$ though it too is known to be weak in a different sense: If $\mathcal{T}$ is a first-order theory, then adding predicative comprehension to $\mathcal{T}$ yields only a conservative extension of $\mathcal{T}$.

\section{Closing Remarks}

In closing, let me emphasize a point that has already been noted a couple times in passing, namely, that the concept of a natural number has played no role whatsoever in this paper. ${ }^{43}$ A critic might seize upon this fact and suggest that the results proven here must therefore be of little significance, since they do not tell us anything about our knowledge of the natural numbers. But I have come to believe that the emphasis one finds, in most philosophical discussions of the concept of number, on the natural numbers may be misplaced. ${ }^{44}$ It is particularly misplaced in discussions of Fregean arithmetics. HP is meant to be the fundamental principle of a theory of cardinal numbers: not of finite cardinal numbers, but of cardinal numbers generally. If so, then our first question should be: What sort of theory of cardinal numbers can we get out of HP? If the answer is "Something akin to Q", then that might well seem exactly right, since $Q$ is a very natural, if elementary, theory of cardinal numbers (Boolos et al., 2007, p. 216).

As the results here make clear, but as should have been clear all along, someone could have quite a lot of knowledge about cardinal numbers without even having the concept of a natural number. Indeed, the concept of a natural number-that is, the concept of finitude-is really very sophisticated. I am not at all sure that most of the undergraduates I have been privileged to teach have had more than a very tenuous grasp of it. Sure, they can wave their hands, but what do they really know about finitude?

The fact that the existence of sums and products is (probably) unprovable in any predicative form of Frege arithmetic thus leaves me unfazed. Do ordinary folk really know that sums always exist? The claim that sums always exist is very strong indeed, being equivalent to the claim

\footnotetext{
${ }^{42}$ This follows from the fact that, if $\mathcal{T}$ is finitely axiomatized and sequential, then $\mathcal{T}$ plus predicative second-order logic interprets $Q+\operatorname{Con}(\mathcal{T})$ (Visser, 2009a, theorem 4.1), which is not interpretable in $\mathcal{T}$, by a version of the second incompleteness theorem due to Pudlák (1985).

${ }^{43}$ Earlier work establishing that various weak Fregean theories interpret Robinson arithmetic does not make use of a concept of natural number, either, in a sense. But those arguments generally have involved restricting the domain to something smaller than the numbers, and this is typically done using either the ancestral or the method of shortening cuts. In either case, it does still look as if something like the concept of natural number is playing a role.

${ }^{44}$ One might make similar criticisms of some of the psychological literature, e.g., Rips et al. (2008).
} 
that the universe can be partitioned into two parts that are equinumerous both with each other and with the universe itself. ${ }^{45}$ What about the claim that natural numbers always have sums? It takes a lot of conceptual sophistication, or so I am suggesting, even properly to grasp that statement, so it does not bother me if it is no part of our 'ordinary mathematical knowledge', that is, if resources well beyond those necessary for 'everyday' arithmetic have to be recruited for a proper proof of it, or even for a proper formulation of it. It is perfectly possible, it seems to me, that our knowledge of the most elementary parts of arithmetic should be founded on predicative FA, but that our knowledge of other parts of arithmetic should depend upon more sophisticated forms of reasoning.

The results proven here show, then, that predicative FA, and even adjunctive FA, are sufficient to ground a substantial fragment of 'everyday' arithmetic. Whether that establishes some form of logicism is, of course, another question. But one should not discount that prospect simply because it concerns only a small fragment of arithmetic. Logicism for $\mathrm{R}^{\prime}$ and $\mathrm{Q}_{R}^{h}$ would still be signficant results, if only because both theories commit us to the existence of infinitely many numbers.

It is another question still whether the arithmetic knowledge of ordinary folk is, in some sense, actually based upon HP. But that is not a question for philosophers to adjudicate. ${ }^{46}$

\section{REFERENCES}

Boolos, G. (1998a). 'The consistency of Frege's Foundations of Arithmetic', in Boolos 1998c, 183-202.

— (1998b). 'Is Hume's principle analytic?', in Boolos 1998c, 301-14.

- (1998c). Logic, Logic, and Logic, Jeffrey, R., ed. Cambridge MA, Harvard University Press.

- (1998d). 'On the proof of Frege's theorem', in Boolos 1998c, 275-91. Boolos, G. and Heck, R. G. (1998). 'Die Grundlagen der Arithmetik $\S \S 82-83$ ', in Boolos 1998c, 315-38.

Boolos, G. S., Burgess, J. P., and Jeffrey, R. C. (2007). Computability and Logic, 5th edition. Cambridge, Cambridge University Press.

\footnotetext{
${ }^{45}$ One direction follows simply by consideration of $\mathrm{N} x:(x=x)+\mathrm{N} x:(x=x)$. The other follows from the fact that, if there is such a partition, then we can always find the sum of $\mathrm{N} x: F x$ and $\mathrm{N} x: G x$ by looking at their images within those partitions (Heck, $2012, \S 10.3$ ). Compare the use Visser (2011) makes of the theory he calls COPY.

${ }^{46} \mathrm{~A}$ talk based on this paper was presented at a conference on the Mathematics of Abstraction, held at the University of London in March 2012 and sponsored by Øystein Linnebo's project on Plurals, Predicates, and Paradox, which is funded by the European Research Council. Thanks to John P. Burgess, Paul Pietroski, and Albert Visser for comments and encouragement early on, and to everyone at the conference for their comments and questions, especially Richard Kaye and Sean Walsh, as well as Øystein and Albert. Extra special thanks to an anonymous referee, whose comments led me to investigate the questions discussed in Section 7 and so greatly improved the paper.
} 
Burgess, J. P. (2005). Fixing Frege. Princeton NJ, Princeton University Press.

Cobham, A. (1960). 'Effectively decidable theories', in A. Tarski et al. (eds.), Summaries of Talks Presented at the Summer Institute for Symbolic Logic, Cornell University, 1957, 2d edition. Princeton, Communications Research Division, Institute for Defense Analyses, 391-5.

Decock, L. (2008). 'Neo-Fregeanism naturalized: The role of one-one correspondence in numerical cognition', Brain and Behavioral Sciences 31: 648-9.

Hájek, P. (2007). 'Mathematical fuzzy logic and natural numbers', Fundamenta Informaticae 81: 155-63.

Hájek, P. and Pudlák, P. (1993). Metamathematics of First-order Arithmetic. New York, Springer-Verlag.

Hazen, A. (1985). 'Review of Crispin Wright, Frege's Conception of Numbers as Objects', Australasian Journal of Philosophy 63: 251-4.

Heck, R. G. (2011a). 'Cardinality, counting, and equinumerosity', in Heck 2011c, 156-79.

- (2011b). 'Finitude and Hume's Principle', in Heck 2011c, 237-60.

- (2011c). Frege's Theorem. Oxford, Clarendon Press.

— (2011d). 'A logic for Frege's Theorem', in Heck 2011c, 267-96.

- (2011e). 'Ramified Frege arithmetic', Journal of Philosophical Logic 40: 715-35.

- (2012). Reading Frege's Grundgesetze. Oxford, Clarendon Press.

Jones, J. P. and Shepherdson, J. C. (1983). 'Variants of Robinson's essentially undecidable theory R', Archiv für mathematische Logik und Grundlagenforschung 23: 61-4.

Linnebo, Ø. (2004). 'Predicative fragments of Frege arithmetic', Bulletin of Symbolic Logic 10: 153-74.

Pietroski, P. and Lidz, J. (2008). 'Natural number concepts: No derivation without formalization', Brain and Behavioral Sciences 31: 666-7.

Pudlák, P. (1985). 'Cuts, consistency statements and interpretations', Journal of Symbolic Logic 50: 423-41.

Rips, L. J., Bloomfield, A., and Asmuth, J. (2008). 'From numerical concepts to concepts of number', Brain and Behavioral Sciences 31: 623-41.

Robinson, J. (1949). 'Definability and decision problems in arithmetic', Journal of Symbolic Logic 14: 98-114.

Švejdar, V. (2007). 'An interpretation of Robinson arithmetic in Grzegorczyk's weaker variant', Fundamenta Informaticae 81: 347-54.

Tarski, A., Mostowski, A., and Robinson, A. (1953). Undecidable Theories. Amsterdam, North-Holland Publishing.

Visser, A. (2009a). 'Can we make the second incompleteness theorem coordinate free?', Journal of Logic and Computation 21: 543-60.

- (2009b). 'Cardinal arithmetic in the style of Baron von Münchhausen', Review of Symbolic Logic 2: 570-89. 
(2009c). 'Why the theory $\mathrm{R}$ is special', in Foundational Adventures: Essays in Honor of Harvey M. Friedman, ed. by N. Tennant. http://foundationaladventures.files.wordpress . com/2012/01/onrfin.pdf.

(2011). 'Hume's principle, beginnings', Review of Symbolic Logic 4: 114-29.

Wright, C. (1983). Frege's Conception of Numbers as Objects. Aberdeen, Aberdeen University Press.

- (2001). 'On the philosophical significance of Frege's theorem', in The Reason's Proper Study. Oxford, Clarendon Press, 272-306.

Department of Philosophy, Box 1918, Brown University, Providence RI 02912 